\title{
LONG TIME UNIFORM STABILITY FOR SOLUTIONS OF $n$-DIMENSIONAL NAVIER-STOKES EQUATIONS
}

\author{
BY \\ LINGHAI ZHANG \\ Department of Mathematics, The Ohio State University, Columbus, Ohio 43210-1174
}

1. Introduction and Main Results. Recently, there has been much attention to stability of the solutions to the Navier-Stokes equations. Recent papers that consider closely related topics are [Se, VS, W2]. Secchi [Se] utilized energy methods to prove his $L^{2}$-stability result for the 3-dimensional case. Veiga and Secchi [VS] and Wiegner [W2] studied stability in $L^{p}(p>n)$ for strong solutions to the Cauchy problem. All these papers establish very interesting results for $n(\geq 3)$-dimensional problems, without employing any kinds of smallness hypotheses. Here are their stability results.

Proposition 1 ([Se]). Let $u_{0} \in H^{1}, \nabla \cdot u_{0}=0, f \in L^{1}\left(0, \infty ; L^{2}\right) \cap L^{2}\left(0, \infty ; L^{2}\right)$, $u \in H^{1}\left(0, \infty ; L^{2}\right) \cap L^{2}\left(0, \infty ; H^{2}\right)$. Then for each $v_{0} \in H$, there is a weak solution corresponding to the initial velocity $v_{0}$ and the external force $f$, such that $\|u(t)-v(t)\| \rightarrow 0$, $t \rightarrow \infty$, where $V=\left\{\phi \mid \operatorname{div} \phi=0, \phi \in L^{2}\right\}$ and $H$ is the $L^{2}$-closure of $V$.

Proposition 2 ([VS]). Let $p>3, a_{1} \in L^{1} \cap L^{p+2}, a_{2} \in L^{1} \cap L^{p}, \nabla \cdot a_{1}=\nabla \cdot a_{2}=0$ and let $u_{1} \in L^{\infty}\left(0, \infty ; L^{p+2}\right)$ be a strong solution corresponding to $a_{1}$. Then there is a constant $\delta>0$, such that if $\left\|a_{1}-a_{2}\right\|_{L^{p}}<\delta$, then there exists a unique solution $u_{2} \in C\left(0, \infty, L^{p}\right)$ with initial data $a_{2}$, and

$$
\left\|u_{1}(t)-u_{2}(t)\right\|_{L^{p}} \leq C(1+t)^{-3 / 4},
$$

where $\delta, C$ depend on $p, L^{1}, L^{p}$ norms of $a_{1}, a_{2}$ and on the $L^{\infty}\left(0, \infty ; L^{p+2}\right)$ norm of $u_{1}$. Proposition 3 ([W2]). Let $p>n \geq 3, n / p+2 / q=1, a_{1}, a_{2} \in L^{2} \cap L^{p}, \nabla \cdot a_{1}=\nabla \cdot a_{2}=0$ and let $u_{1} \in L^{q}\left(0, \infty ; L^{p}\right)$ be a strong solution corresponding to $a_{1}$. Then there exists a constant $\delta>0$ such that if $\left\|a_{1}-a_{2}\right\|_{L^{p}}<\delta$, then a strong solution $u_{2}$ exists with $u_{2} \in L^{r}\left(0, \infty ; L^{p}\right)$ and $4 p /(n(p-2))<r \leq \infty$.

In [V], Veiga proved that if a global solution of the $n$-dimensional problem fulfills $u \in L^{q}\left(0, \infty ; L^{p}\right)$, then $u \in L^{\infty}\left(0, \infty ; L^{p}\right)$, where $u_{0} \in L^{2} \cap L^{p}, p>n \geq 3, n / p+2 / q=$

Received November 9, 1996.

1991 Mathematics Subject Classification. Primary 35Q10, 35Q20.

Key words and phrases. Long time uniform stability, Navier-Stokes equations, integral estimation.

This work was partially done when the author was at Beijing Institute of Applied Physics and Computational Mathematics in 1993. 
1. Unfortunately, the existence of a solution in the class $L^{q}\left(0, \infty ; L^{p}\right)$ is still an open problem for general data $u_{0}$, but is at least valid for Wiegner's initial condition. In [W3] he proved that if $u_{0} \in L^{1} \cap L^{p}, p>n,(1+|x|) u_{0}(x) \in L^{1}, \int_{R^{n}} u_{0}(x) d x=0$, then $\|u(t)\|_{\infty}=O\left(t^{-(n+1) / 2}\right)$.

For the 2-dimensional problem, it has been established that for any initial data $u_{0} \in$ $H^{2}$, there is a unique global strong solution. For the $n(\geq 3)$-dimensional case, it is known that for small initial data, there exists a global strong solution. However, we can utilize Veiga's hypotheses rather than the smallness condition to prove the uniform stability. Then by utilizing Wiegner's $L^{\infty}$-decay result [W3], we can obtain the uniform stability for pressure $p$.

In this note we study long time uniform stability with respect to perturbation of the initial velocity for solutions to the Cauchy problem for the $n(\geq 2)$-dimensional incompressible Navier-Stokes equations (NSE)

$$
\begin{gathered}
u_{t}+u \cdot \nabla u-\Delta u+\nabla p=0, \quad \nabla \cdot u=0, \\
u(x, 0)=u_{0}(x), \quad \nabla \cdot u_{0}=0,
\end{gathered}
$$

where $x=\left(x_{1}, \ldots, x_{n}\right) \in R^{n}, t>0$ are spatial and temporal variables, $u=\left(u_{1}(x, t), \ldots\right.$, $\left.u_{n}(x, t)\right) \in R^{n}, p=p(x, t)$ denote the unknown velocity and pressure respectively, $u_{t}=$ $\frac{\partial u}{\partial t}, \nabla$ and $\Delta$ are gradient and Laplace operators respectively.

We also study long time asymptotic behavior of the solutions for the 2-dimensional case and regularity for the $n(\geq 3)$-dimensional problem. In the whole paper, we assume that $u_{0}$ and $(u, p)$ of problem (1-2) satisfy $\nabla \cdot u_{0}=\nabla \cdot u=0$ and $\lim _{|x| \rightarrow \infty} p(x, t)=$ $\lim _{|x| \rightarrow \infty}\left|\frac{\partial^{\alpha_{1}+\cdots+\alpha_{n}} u(x, t)}{\partial x_{1}^{\alpha_{1}} \cdots \partial x_{n}^{\alpha_{n}}}\right|=0$, where $\alpha_{1}, \ldots, \alpha_{n}$ are integers, $\alpha_{1}+\cdots+\alpha_{n} \leq 2, t \geq 0$.

Lemma 1.1. Let $n=2, u_{0} \in H^{2}$. Then problem (1-2) enjoys a unique global solution $(u, p) \in L^{\infty}\left(0, T ; H^{2}\right) \cap W^{1, \infty}\left(0, T ; L^{2}\right)$, where $T>0$ is any finite constant.

Lemma 1.1 can be proved by the fixed point principle together with some of the estimates displayed in Lemmas 2.2, 3.1-3.4.

Let $(u, p)$ and $(v, q)$ be the solutions of problem (1-2) corresponding to $u_{0}, v_{0}$. Let $(w, \pi)=(u-v, p-q)$. Then they satisfy the equations

$$
\begin{array}{cc}
w_{t}+w \cdot \nabla u+v \cdot \nabla w-\Delta w+\nabla \pi=0, & \nabla \cdot w=0, \\
w(x, 0)=w_{0}(x)=u_{0}(x)-v_{0}(x), & \nabla \cdot w_{0}=0 .
\end{array}
$$

Definition. If there exists a constant $C>0$, which may depend on $\left\|u_{0}\right\|_{X},\left\|v_{0}\right\|_{X}$, but which does not depend on $t \geq 0$, such that

$$
(1+t)^{\alpha}\|(w, \pi)(t)\|_{Y} \leq C\left\|w_{0}\right\|_{X}, \quad \text { for any } 0 \leq t<\infty,
$$

then the solutions of problem (1-2) are called uniformly stable, where $X, Y$ are certain Sobolev spaces and $\alpha \geq 0$ is a constant.

It is very interesting and important that the upper bounds of certain norms of $(w, \pi)$ depend explicitly on $u_{0}-v_{0}$, but do not depend on $t \geq 0$. Although it is very difficult to study the uniform stability of the solutions to problem (1-2), we can at least establish the best rates of decay in different Sobolev spaces respectively for the 2-dimensional problem. 
Many mathematicians have studied the asymptotic behavior of solutions to (1) and have made significant progress in this area [H, K, KM, W1, W2, W3, S, V, Z3]. They verified that solutions to the $n(\geq 2)$-dimensional problem have the same rates of decay as those of the heat equations, provided the initial velocities are in the same class.

The decay estimates do not automatically lead to the uniform stability of the solutions to (1). In fact, solutions to the $n(\geq 3)$-dimensional problem have the decay estimate $\|u(t)\| \leq C(1+t)^{-n / 4}$, if $u_{0} \in L^{1} \cap H^{2}$. But if $u \notin L^{q}\left(0, \infty ; L^{p}\right)$, i.e., $\int_{0}^{\infty}\|u(t)\|_{L^{p}}^{q} d t=\infty$, where $p>n \geq 3, n / p+2 / q=1$, nobody has proved the uniform stability yet. If $n=2$, the proof of the uniform $L^{2}$-stability $\|w(t)\| \leq C\left\|w_{0}\right\|$, where $C$ is a constant depending only on $\left\|u_{0}\right\|$, relies only on some elaborate energy estimate, i.e., $\|u(t)\| \leq\left\|u_{0}\right\|,\|\nabla u(t)\|^{2} \in$ $L^{1}[0, \infty)$. If $n \geq 3$, the proof depends only on the assumption $u_{0} \in L^{2} \cap L^{p}, u \in$ $L^{q}\left[0, \infty ; L^{p}\right), p>n \geq 3, n / p+2 / q=1$. It can be clearly seen that the establishment of this elementary stability is quite independent of all the asymptotic behaviors. We do not necessarily require that any norm of the solution of problem (1-2) tends to zero as $t \rightarrow \infty$ to justify this basic uniform stability. On the other hand, the algebraic rates of decay suggest that certain norms of $w$ have some algebraic rates of decay.

We will study the optimal decay rates of various norms of $(w, \pi)$ corresponding to $w_{0}$, which may be in the Sobolev spaces $H^{2}$ or $L^{1} \cap H^{2}$. We employ the method of energy estimation. We also employ various inequalities. The comprehensive use of Hölder's inequality, Gagliardo-Nirenberg's inequality, Gronwall's inequality and the relation $\nabla$. $u=0$ is very important and is used almost everywhere to establish the $L^{2}$ and $L^{\infty}$ long time uniform stability.

For the 2-dimensional problem (1-2), if $u_{0} \in L^{1} \cap H^{2}$, Wiegner [W1] obtained

$$
(1+t)^{1 / 2}\|u(t)\|+(1+t)^{3 / 2}\|\Delta u(t)\|+(1+t)\|u(t)\|_{\infty} \leq C .
$$

If $u_{0} \in L^{1} \cap H^{2}, \int_{R^{2}} u_{0} d x \neq 0$, then

$$
0<C_{1} \leq(1+t)^{1 / 2}\|u(t)\| \leq C_{2}<\infty .
$$

For the 2-dimensional problem (3-4), we have similar results to these displayed decay estimates.

Denote by $C$ any positive constant appearing in our paper, which may be different from line to line, and which may depend on the $L^{1}, L^{2}$ and $H^{2}$ norms of $u_{0}, v_{0}$, but which never depends on $t \geq 0$. Moreover, denote by $L^{p}=L^{p}\left(R^{n}\right), L^{\infty}=L^{\infty}\left(R^{n}\right)$, $H^{m}=H^{m}\left(R^{n}\right), L=L^{1}\left(R^{n}\right) \cap L^{2}\left(R^{n}\right), H=L^{1}\left(R^{n}\right) \cap H^{2}\left(R^{n}\right)$, where $p, m \geq 1$ are integers, and denote by

$$
\begin{gathered}
\int_{R^{n}} u(x) d x=\int_{R^{n}} u\left(x_{1}, \ldots, x_{n}\right) d x_{1} \cdots d x_{n}, \quad|\nabla u|^{2}=\sum_{i=1}^{n}\left|\nabla u_{i}\right|^{2}, \\
\|u(t)\|=\|u(t)\|_{L^{2}}, \quad\|u(t)\|_{\infty}=\|u(t)\|_{L^{\infty}}, \quad\|u(t)\|_{m}=\|u(t)\|_{H^{m}} \\
\left\|w_{0}\right\|_{L}=\left\|w_{0}\right\|_{L^{1}}+\left\|w_{0}\right\|_{L^{2}}, \quad\left\|w_{0}\right\|_{H}=\left\|w_{0}\right\|_{L^{1}}+\left\|w_{0}\right\|_{H^{2}} .
\end{gathered}
$$

For a weighted Sobolev space $W$, denote by

$$
\|u\|_{W}=\int_{R^{n}}|x||u(x)| d x, \quad\left\|w_{0}\right\|_{M}=\left\|w_{0}\right\|_{W}+\left\|w_{0}\right\|_{L^{1}}+\left\|w_{0}\right\|_{H^{2}}
$$


Let $f(x) \in L^{1} \cap L^{2}$ and define its Fourier transform

$$
F[f](\xi)=\hat{f}(\xi)=\int_{R^{n}} f(x) \exp (-i x \cdot \xi) d x .
$$

The definition can be extended to the Hilbert space $L^{2}$ by continuity.

In Section 2, we present a series of elementary estimates relating to problem (12 ) or (3-4). Some other inequalities are also listed here. Section 3 is dedicated to temporal asymptotic behavior of solutions of the 2-dimensional problem (1-2) for the case $u_{0} \in H^{2}$. After having established all the necessary asymptotic behaviors with $u_{0} \in H^{2}$, we can prove our uniform $L^{2}$ and $L^{\infty}$ stability for the 2-dimensional problem (1-2) with $u_{0}, v_{0} \in H^{2}$ and $L^{1} \cap H^{2}$. This is done in Section 4. In Section 5, we deal with $n(\geq 3)$-dimensional problems. We find that if $u_{0} \in L^{2} \cap L^{p}, u \in L^{q}\left(0, \infty ; L^{p}\right)$, for some $p>n \geq 3, n / p+2 / q=1$, then $u$ has more regularity, namely

$$
u \in\left(\bigcap_{p \leq r<\infty} L^{\frac{2 r}{r-n}}\left(0, \infty ; L^{r}\right)\right) \cap\left(\bigcap_{2 \leq s<\infty} L^{\infty}\left(0, \infty ; L^{2}\right)\right) .
$$

Let us state our long time uniform stability theorems.

ThEOREM 1. Let $(u, p)$ and $(v, q)$ be the solutions of the 2-dimensional problem (1-2) corresponding to the initial velocities $u_{0}$ and $v_{0}$. Let $(w, \pi)=(u-v, p-q)$. If $u_{0}, v_{0} \in H^{2}$, then we have the uniform stability estimate

$$
\begin{aligned}
\|w(t)\| & +(1+t)^{1 / 2}\|\nabla w(t)\|+(1+t)\|\Delta w(t)\| \\
& +(1+t)^{1 / 2}\|w(t)\|_{\infty}+(1+t)\left\|w_{t}(t)\right\|+(1+t)^{1 / 2}\|\pi(t)\| \\
& +(1+t)\|\nabla \pi(t)\|+(1+t)^{3 / 2}\|\Delta \pi(t)\| \\
& +(1+t)\|\pi(t)\|_{\infty}+(1+t)^{3 / 2}\left\|\pi_{t}(t)\right\| \leq C\left\|w_{0}\right\|_{H} .
\end{aligned}
$$

If $u_{0}, v_{0} \in H^{2}, w_{0} \in L^{1} \cap H^{2},\|u(t)\|+\|v(t)\| \leq C / \ln (e+t)$, then we have the uniform estimate

$$
\begin{gathered}
(1+t)^{1 / 2}\left\{\|w(t)\|+(1+t)^{1 / 2}\|\nabla w(t)\|+(1+t)\|\Delta w(t)\|\right. \\
+(1+t)^{1 / 2}\|w(t)\|_{\infty}+(1+t)\left\|w_{t}(t)\right\|+(1+t)^{1 / 2}\|\pi(t)\| \\
+(1+t)\|\nabla \pi(t)\|+(1+t)^{3 / 2}\|\Delta \pi(t)\| \\
\left.+(1+t)\|\pi(t)\|_{\infty}+(1+t)^{3 / 2}\left\|\pi_{t}(t)\right\|\right\} \leq C\left\|w_{0}\right\|_{H} .
\end{gathered}
$$

If $u_{0}, v_{0} \in L^{1} \cap H^{2}, \int_{R^{2}} w_{0} d x \neq 0$, we also have the lower bound uniform stability estimate

$$
\begin{gathered}
C_{5}\left|\int_{R^{2}} w_{0} d x\right|-\frac{C_{6} \ln (1+t)}{\sqrt{1+t}}\left\|w_{0}\right\|_{L} \leq(1+t)^{1 / 2}\|w(t)\| \leq C_{7}\left\|w_{0}\right\|_{L}, \\
C_{8}\left|\int_{R^{2}} w_{0} d x\right|-\frac{C_{9} \ln (1+t)}{\sqrt{1+t}}\left\|w_{0}\right\|_{H} \leq(1+t)^{3 / 2}\|w(t)\| \leq C_{10}\left\|w_{0}\right\|_{H} .
\end{gathered}
$$


If $u_{0}, v_{0} \in L^{1} \cap H^{2}, \int_{R^{2}} w_{0} d x=0, \int_{R^{2}}|x|\left|w_{0}\right| d x<\infty$, then we have the uniform stability estimate

$$
\begin{gathered}
(1+t)\left\{\|w(t)\|+(1+t)^{1 / 2}\|\nabla w(t)\|+(1+t)\|\Delta w(t)\|\right. \\
+(1+t)^{1 / 2}\|w(t)\|_{\infty}+(1+t)\left\|w_{t}(t)\right\|+(1+t)^{1 / 2}\|\pi(t)\| \\
+(1+t)\|\nabla \pi(t)\|+(1+t)^{3 / 2}\|\Delta \pi(t)\| \\
\left.\quad+(1+t)\|\pi(t)\|_{\infty}+(1+t)^{3 / 2}\left\|\pi_{t}(t)\right\|\right\} \leq C\left\|w_{0}\right\|_{M} .
\end{gathered}
$$

THEOREM 2. Let $(u, p)$ and $(v, q)$ be the weak solutions of the $n(\geq 3)$-dimensional problem (1-2) corresponding to the initial data $u_{0}$ and $v_{0}$. Let $(w, \pi)=(u-v, p-q)$. If $u_{0} \in L^{2} \cap L^{p}, u \in L^{q}\left(0, \infty ; L^{p}\right)$, for some $p>n \geq 3, n / p+2 / q=1$, then $u \in L^{\infty}\left(0, \infty ; L^{p}\right)$. If $v_{0} \in L^{2}$, we also have the uniform stability estimate

$$
\|w(t)\| \leq C\left\|w_{0}\right\|, \quad \int_{0}^{\infty}\|\nabla w(t)\|^{2} d t \leq C\left\|w_{0}\right\|^{2}, \quad\|\hat{\pi}(t)\|_{\infty} \leq C\left\|w_{0}\right\|,
$$

where constant $C$ depends only on the $L^{q}\left(0, \infty ; L^{p}\right)$ norm of $u$.

If $u_{0}, v_{0} \in L^{2} \cap L^{p}, u \in L^{q}\left(0, \infty ; L^{p}\right)$, for some $p>n \geq 3, n / p+2 / q=1$, then there exists a constant $\delta>0$, such that if $\left\|u_{0}-v_{0}\right\|_{L^{p}}<\delta$, a strong solution $v$ exists with $v \in L^{r}\left(0, \infty ; L^{p}\right), 4 p /(n(p-2))<r \leq \infty$, and

$$
\begin{gathered}
\sup _{\left\|u_{0}-v_{0}\right\|_{L^{p}}<\delta} \int_{0}^{\infty}\|v(t)\|_{L^{p}}^{q} d t \leq C<\infty \\
\|u(t)-v(t)\|_{L^{p}} \leq C\left\|u_{0}-v_{0}\right\|_{L^{p}} \\
\int_{0}^{\infty} \int_{R^{n}}|u-v|^{p-2}|\nabla(u-v)|^{2} d x d t+\int_{0}^{\infty} \int_{R^{n}}\left|\nabla\left(|u-v|^{p / 2}\right)\right|^{2} d x d t \leq C\left\|u_{0}-v_{0}\right\|_{L^{p}}^{p},
\end{gathered}
$$

where $C$ depends on $\int_{0}^{\infty}\|u(t)\|_{L^{p}}^{q} d t$.

If $u_{0}, v_{0} \in L^{2} \cap L^{\infty}, u \in L^{q}\left(0, \infty ; L^{p}\right)$, for some $p>n \geq 3, n / p+2 / q=1$, then for any $r \geq p$, there exists a constant $\delta(r, n)>0$, such that if $\left\|u_{0}-v_{0}\right\|_{L^{r}}<\delta(r, n)$, then

$$
\begin{gathered}
\|u(t)-v(t)\|_{L^{r}} \leq C(n, r)\left\|u_{0}-v_{0}\right\|_{L^{r}}, \\
\int_{0}^{\infty} \int_{R^{n}}|u-v|^{r-2}|\nabla(u-v)|^{2} d x d t+\int_{0}^{\infty} \int_{R^{n}}\left|\nabla\left(|u-v|^{r / 2}\right)\right|^{2} d x d t \leq C\left\|u_{0}-v_{0}\right\|_{L^{r}}^{r},
\end{gathered}
$$

where $C(n, r)$ depends on the norm $\int_{0}^{\infty}\|u(t)\|_{L^{r}}^{2 r /(r-n)} d t$. then

If $u_{0}, v_{0} \in L^{1} \cap L^{p},(1+|x|)\left[\left|u_{0}(x)\right|+\left|v_{0}(x)\right|\right] \in L^{1}, \int_{R^{n}} u_{0}(x) d x=\int_{R^{n}} v_{0}(x) d x=0$,

$$
t^{(n+1) / 2}\|\pi(t)\| \leq C\left\|w_{0}\right\| .
$$

TheOREM 3. Let $(u, p)$ be the solutions of the 2-dimensional problem (1-2) corresponding to the initial velocity $u_{0}$. Then we have the decay estimates for the solutions $(u, p)$

$$
\begin{gathered}
\lim _{t \rightarrow \infty}\left[(1+t)\|\nabla u(t)\|^{2}\right]=0, \\
\lim _{t \rightarrow \infty}\left[(1+t)\|p(t)\|^{2}+(1+t)^{2}\|\nabla p(t)\|^{2}\right]=0,
\end{gathered}
$$




$$
\begin{aligned}
(1+t)^{\beta}\{\| & u(t)\left\|+(1+t)^{1 / 2}\right\| \nabla u(t) \| \\
& +(1+t)\|\Delta u(t)\|+(1+t)^{1 / 2}\|u(t)\|_{\infty} \\
& +(1+t)\left\|u_{t}(t)\right\|+(1+t)^{1 / 2}\|p(t)\| \\
& +(1+t)\|\nabla p(t)\|+(1+t)^{3 / 2}\|\Delta p(t)\| \\
& \left.+(1+t)\|p(t)\|_{\infty}+(1+t)^{3 / 2}\left\|p_{t}(t)\right\|\right\} \leq C .
\end{aligned}
$$

If $u_{0} \in H^{2}$, then $\beta=0$. If $u_{0} \in L^{1} \cap H^{2}$, then $\beta=1 / 2$. If $u_{0} \in L^{1} \cap H^{2}, \int_{R^{2}} u_{0} d x=0$, $\int_{R^{2}}|x|\left|u_{0}\right| d x<\infty$, then $\beta=1$. If $u_{0} \in H^{3}$, then $\beta=0$. We also have

$$
\begin{aligned}
& (1+t)^{3 / 2}\|\nabla \Delta u(t)\|+(1+t)\|\nabla u(t)\|_{\infty} \\
& \quad+(1+t)^{3 / 2}\left\|\nabla u_{t}(t)\right\|+(1+t)^{2}\left\|\nabla p_{t}(t)\right\| \leq C .
\end{aligned}
$$

TheOREm 4. Let $u_{0} \in L^{2} \cap L^{\infty}, u \in L^{q}\left(0, \infty ; L^{p}\right)$, for some $p>n \geq 3, n / p+2 / q=1$. Then $u \in\left(\bigcap_{p \leq r<\infty} L^{2 r /(r-n)}\left(0, \infty ; L^{r}\right)\right) \cap\left(\bigcap_{2 \leq s<\infty} L^{\infty}\left(0, \infty ; L^{s}\right)\right)$.

2. Elementary Estimates. In this section we present some elementary estimates that will be very useful in demonstrating our uniform stability.

LEMmA 2.1. The following preliminary estimates are valid for the 2-dimensional problem:

$$
\begin{gathered}
|F[w \cdot \nabla u]| \leq|\xi|\|u(t)\|\|w(t)\|, \\
|\hat{\pi}| \leq[\|u(t)\|+\|v(t)\|]\|w(t)\|, \\
\|\pi(t)\| \leq\left[\|u(t)\|_{\infty}+\|v(t)\|_{\infty}\right]\|w(t)\|, \\
\|\Delta \pi(t)\| \leq C\left[\|\Delta u(t)\|^{1 / 2}+\|\Delta v(t)\|^{1 / 2}\right]\|\Delta w(t)\| \\
+C\left[\|\Delta u(t)\|^{3 / 2}+\|\Delta v(t)\|^{3 / 2}\right]\|w(t)\|, \\
\left\|w_{t}(t)\right\| \leq\|w(t)\|_{\infty}\|\nabla u(t)\| \\
+\|v(t)\|_{\infty}\|\nabla w(t)\|+\|\Delta w(t)\|+\|\nabla \pi(t)\|, \\
\left\|\pi_{t}(t)\right\| \leq\left\|w_{t}(t)\right\|\|u(t)\|_{\infty}+\|w(t)\|_{\infty}\left\|u_{t}(t)\right\|, \\
\int_{R^{2}} f \cdot(g \cdot \nabla f) d x=0, \quad \int_{R^{2}}[f \cdot(g \cdot \nabla h)+h \cdot(g \cdot \nabla f)] d x=0, \\
\left|\int_{R^{2}} f \cdot(g \cdot \nabla h) d x\right| \leq \int_{R^{2}}|g||h||\nabla f| d x
\end{gathered}
$$

where $f, g, h \in H^{1}$ with $\nabla \cdot g=0$ are arbitrary vector-valued functions.

The first two estimates are necessary to bound $|\hat{w}|$ in Lemma 2.3, which is a very important step for obtaining some of the main results. See Lemmas 4.2, 4.3, and 4.4. Some other estimates follow by taking the divergence of (1) and integrating the results appropriately. They play significant roles in establishing Theorem 1. The last one is important in some simplifications. The first three and the last two estimations are also valid for the $n$-dimensional case, $n \geq 2$. 
Proof. It is easy to get the following identities by using $\nabla \cdot w=0$ :

$$
\begin{gathered}
w \cdot \nabla u=\left(\sum_{j=1}^{n} \frac{\partial}{\partial x_{j}}\left(u_{i} w_{j}\right)\right)_{1 \leq i \leq n}, \\
F[w \cdot \nabla u]=\sqrt{-1}\left(\sum_{j=1}^{n} \xi_{j} \widehat{u_{j} w_{j}}\right)_{1 \leq i \leq n} .
\end{gathered}
$$

Thus we get

$$
\begin{aligned}
|F[w \cdot \nabla u]|^{2} & =\sum_{i=1}^{n}\left|\sum_{j=1}^{n} \xi_{j} \widehat{u_{i} w_{j}}\right|^{2} \leq \sum_{i=1}^{n}\left(\sum_{j=1}^{n} \xi_{j}^{2}\right)\left(\sum_{j=1}^{n}\left|\widehat{u_{i} w_{j}}\right|^{2}\right) \\
& \leq|\xi|^{2} \sum_{i=1}^{n} \sum_{j=1}^{n}\left\|u_{i}(t)\right\|^{2}\left\|w_{j}(t)\right\|^{2}=|\xi|^{2}\|u(t)\|^{2}\|w(t)\|^{2}
\end{aligned}
$$

where we have used $|\widehat{f g}| \leq\|f(t)\|\|g(t)\|$.

The following identity follows from (1):

$$
\Delta p=-\sum_{i=1}^{n} \sum_{j=1}^{n} \frac{\partial^{2}}{\partial x_{i} \partial x_{j}}\left(u_{i} u_{j}\right) .
$$

The Fourier transform yields

$$
|\xi|^{2} \hat{p}=-\sum_{i=1}^{n} \sum_{j=1}^{n} \xi_{i} \xi_{j} \widehat{u_{i} u_{j}}
$$

Thus we get the identity

$$
|\xi|^{2} \hat{\pi}=-\sum_{i=1}^{n} \sum_{j=1}^{n} \xi_{i} \xi_{j}\left(\widehat{w_{i} u_{j}}+\widehat{v_{i} w_{j}}\right)
$$

and the estimates

$$
\begin{aligned}
|\xi|^{2}|\hat{\pi}| & \leq \sum_{i=1}^{n} \sum_{j=1}^{n}\left|\xi_{i}\right|\left|\xi_{j}\right|\left[\left\|w_{i}(t)\right\|\left\|u_{j}(t)\right\|+\left\|v_{i}(t)\right\|\left\|w_{j}(t)\right\|\right] \\
& =\sum_{i=1}^{n}\left|\xi_{i}\right|\left\|w_{i}(t)\right\| \sum_{j=1}^{n}\left|\xi_{j}\right|\left\|u_{j}(t)\right\|+\sum_{i=1}^{n}\left|\xi_{i}\right|\left\|v_{i}(t)\right\| \sum_{j=1}^{n}\left|\xi_{j}\right|\left\|w_{j}(t)\right\| \\
& \leq|\xi|^{2}[\|u(t)\|+\|v(t)\|]\|w(t)\| .
\end{aligned}
$$

Therefore we get

$$
|\hat{\pi}| \leq\|w(t)\|[\|u(t)\|+\|v(t)\|]
$$


We can easily get the identity

$$
\begin{aligned}
|\xi|^{4}|\hat{\pi}|^{2} & =\left|\sum_{i=1}^{n} \sum_{j=1}^{n} \xi_{i} \xi_{j}\left(\widehat{w_{i} u_{j}}+\widehat{v_{i} w_{j}}\right)\right|^{2} \\
& \leq \sum_{i=1}^{n} \sum_{j=1}^{n}\left|\xi_{i} \xi_{j}\right|^{2} \sum_{i=1}^{n} \sum_{j=1}^{n}\left|\widehat{w_{i} u_{j}}+\widehat{v_{i} w_{j}}\right|^{2} \\
& \leq|\xi|^{2} \sum_{i=1}^{n} \sum_{j=1}^{n}\left|\widehat{w_{i} u_{j}}+\widehat{v_{i} w_{j}}\right|^{2}
\end{aligned}
$$

or

$$
|\hat{\pi}|^{<} \leq \sum_{i=1}^{n} \sum_{j=1}^{n}\left|\widehat{w_{i} u_{j}}+\widehat{v_{i} w_{j}}\right|^{2}
$$

Moreover, we have

$$
\begin{aligned}
\|\pi(t)\| & =\|\hat{\pi}(t)\| \leq \sum_{i=1}^{n} \sum_{j=1}^{n}\left\|\left(w_{i} u_{j}+v_{i} w_{j}\right)(t)\right\| \\
& \leq \sum_{i=1}^{n} \sum_{j=1}^{n}\left[\left\|u_{j}(t)\right\|_{\infty}\left\|w_{i}(t)\right\|+\left\|v_{i}(t)\right\|_{\infty}\left\|w_{j}(t)\right\|\right] \\
& \leq\|u(t)\|_{\infty}\|w(t)\|+\|v(t)\|_{\infty}\|w(t)\| \\
& \leq\left[\|u(t)\|_{\infty}+\|v(t)\|_{\infty}\right]\|w(t)\| .
\end{aligned}
$$

Taking the divergence of equations (3) we get

$$
\Delta \pi=-\nabla(w \cdot \nabla u+v \cdot \nabla w) .
$$

By using this identity we get the following:

$$
\begin{aligned}
\|\Delta \pi(t)\|^{2}= & \|\nabla(w \cdot \nabla u+v \cdot \nabla w)(t)\|^{2} \\
\leq & C \int_{R^{2}}|\nabla u|^{2}|\nabla w|^{2} d x+C \int_{R^{2}}|\nabla v|^{2}|\nabla w|^{2} d x \\
& +C\|w(t)\|_{\infty}^{2}\|\Delta u(t)\|^{2}+C\|v(t)\|_{\infty}^{2}\|\Delta w(t)\|^{2}, \\
C \int_{R^{2}}|\nabla u|^{2}|\nabla w|^{2} d x \leq & C\|\nabla u(t)\|_{L^{4}}^{2}\|\nabla w(t)\|_{L^{4}}^{2} \\
\leq & C\|u(t)\|^{1 / 2}\|\Delta u(t)\|^{3 / 2}\|w(t)\|^{1 / 2}\|\Delta w(t)\|^{3 / 2} \\
\leq & C\|\Delta u(t)\|\|\Delta w(t)\|^{2}+C\|u(t)\|^{2}\|\Delta u(t)\|^{3}\|w(t)\|^{2} .
\end{aligned}
$$

Similarly, we have

$$
C \int_{R^{2}}|\nabla v|^{2}|\nabla w|^{2} d x \leq C\|\Delta v(t)\|\|\Delta w(t)\|^{2}+C\|v(t)\|^{2}\|\Delta v(t)\|^{3}\|w(t)\|^{2} .
$$


We also have the estimates

$$
\begin{aligned}
C\|w(t)\|_{\infty}^{2}\|\Delta u(t)\|^{2} & \leq C\|w(t)\|\|\Delta w(t)\|\|\Delta u(t)\|^{2} \\
& \leq C\|\Delta u(t)\|\|\Delta w(t)\|^{2}+C\|\Delta u(t)\|^{3}\|w(t)\|^{2}, \\
C\|v(t)\|_{\infty}^{2}\|\Delta w(t)\|^{2} & \leq C\|v(t)\|\|\Delta v(t)\|\|\Delta w(t)\|^{2}
\end{aligned}
$$

Thus we have

$$
\begin{aligned}
\|\Delta \pi(t)\|^{2} \leq & C[\|\Delta u(t)\|+\|\Delta v(t)\|]\|\Delta w(t)\|^{2} \\
& +C\left[\|\Delta u(t)\|^{3}+\|\Delta v(t)\|^{3}\right]\|w(t)\|^{2} .
\end{aligned}
$$

The following estimates follow directly from equations (3) and $|w \cdot \nabla u| \leq|w||\nabla u|$ :

$$
\begin{aligned}
\left\|w_{t}(t)\right\| & \leq\|(w \cdot \nabla u)(t)\|+\|(v \cdot \nabla w)(t)\|+\|\Delta w(t)\|+\|\nabla \pi(t)\| \\
& \leq\|w(t)\|_{\infty}\|\nabla u(t)\|+\|v(t)\|_{\infty}\|\nabla w(t)\|+\|\Delta w(t)\|+\|\nabla \pi(t)\| .
\end{aligned}
$$

Further, we have

$$
|\xi|^{2} \widehat{\pi}_{t}=-\sum_{i=1}^{2} \sum_{j=1}^{2} \xi_{i} \xi_{j}\left(\widehat{w_{i t} u_{j}}+\widehat{w_{i} u_{j t}}+\widehat{v_{i t} w_{j}}+\widehat{v_{i} w_{j t}}\right)
$$

Thus

$$
\begin{aligned}
|\xi|^{4}\left|\hat{\pi}_{t}\right|^{2} & \leq \sum_{i=1}^{2} \sum_{j=1}^{2}\left|\xi_{i} \xi_{j}\right|^{2} \sum_{i=1}^{2} \sum_{j=1}^{2}\left|\widehat{w_{i t} u_{j}}+\widehat{w_{i} u_{j t}}+\widehat{v_{i t} w_{j}}+\widehat{v_{i} w_{j t}}\right|^{2} \\
& =|\xi|^{4} \sum_{i=1}^{2} \sum_{j=1}^{2}\left|\widehat{w_{i t} u_{j}}+\widehat{w_{i} u_{j t}}+\widehat{v_{i t} w_{j}}+\widehat{v_{i} w_{j t}}\right|^{2}
\end{aligned}
$$

or equivalently, we have

$$
\left|\widehat{\pi}_{t}\right|^{2} \leq \sum_{i=1}^{2} \sum_{j=1}^{2}\left|\widehat{w_{i t} u_{j}}+\widehat{w_{i} u_{j t}}+\widehat{v_{i t} w_{j}}+\widehat{v_{i} w_{j t}}\right|^{2}
$$


Now by utilizing this inequality, we get

$$
\begin{aligned}
\left\|\pi_{t}(t)\right\|= & \left\|\widehat{\pi_{t}}(t)\right\| \\
\leq & \sum_{i=1}^{2} \sum_{j=1}^{2}\left\|\left(\widehat{w_{i t} u_{j}}+\widehat{w_{i} u_{j t}}+\widehat{v_{i t} w_{j}}+\widehat{v_{i} w_{j t}}\right)(t)\right\| \\
= & \sum_{i=1}^{2} \sum_{j=1}^{2}\left\|\left(w_{i t} u_{j}+w_{i} u_{j t}+v_{i t} w_{j}+v_{i} w_{j t}\right)(t)\right\| \\
\leq & \sum_{i=1}^{2} \sum_{j=1}^{2}\left[\left\|u_{j}(t)\right\|_{\infty}\left\|w_{i t}(t)\right\|+\left\|w_{i}(t)\right\|_{\infty}\left\|u_{j t}(t)\right\|\right. \\
\leq & \|u(t)\|_{\infty}\left\|w_{t}(t)\right\|+\|w(t)\|_{\infty}\left\|u_{t}(t)\right\| \\
& +\|w(t)\|_{\infty}\left\|v_{t}(t)\right\|+\|v(t)\|_{\infty}\left\|w_{t}(t)\right\| \\
\leq & \|u(t)\|_{\infty}\left\|w_{t}(t)\right\|+\|w(t)\|_{\infty}\left\|u_{t}(t)\right\| \\
& +\|w(t)\|_{\infty}\left\|\left(v_{t}, B_{t}\right)(t)\right\|+\|v(t)\|_{\infty}\left\|w_{t}(t)\right\| \\
= & \left\|w_{t}(t)\right\|\left[\|u(t)\|_{\infty}+\|v(t)\|_{\infty}\right] \\
& +\|w(t)\|_{\infty}\left[\left\|u_{t}(t)\right\|+\left\|\left(v_{t}, B_{t}\right)(t)\right\|\right] .
\end{aligned}
$$

Since

$$
\begin{aligned}
\int_{R^{n}} f \cdot(g \cdot \nabla h) d x & =\int_{R^{n}} \sum_{i=1}^{n} \sum_{j=1}^{n} f_{i} g_{j} \frac{\partial h_{i}}{\partial x_{j}} d x \\
& =\int_{R^{n}} \sum_{i=1}^{n} \sum_{j=1}^{n} f_{i} \frac{\partial}{\partial x_{j}}\left(g_{j} h_{i}\right) d x \\
& =-\int_{R^{n}} \sum_{i=1}^{n} \sum_{j=1}^{n} g_{j} h_{i} \frac{\partial f_{i}}{\partial x_{j}} d x=-\int_{R^{n}} h \cdot(g \cdot \nabla f) d x
\end{aligned}
$$

we get

$$
\int_{R^{n}} f \cdot(g \cdot \nabla f) d x=0, \quad \int_{R^{n}}[f \cdot(g \cdot \nabla h)+h \cdot(g \cdot \nabla f)] d x=0 .
$$

In addition, since

$$
\left|\sum_{i=1}^{n} \sum_{j=1}^{n} g_{j} h_{i} \frac{\partial f_{i}}{\partial x_{j}}\right| \leq\left(\sum_{i=1}^{n} \sum_{j=1}^{n}\left|g_{j} h_{i}\right|^{2}\right)^{1 / 2}\left(\sum_{i=1}^{n} \sum_{j=1}^{n}\left|\frac{\partial f_{i}}{\partial x_{j}}\right|^{2}\right)^{1 / 2}=|g||h||\nabla f|
$$

we have

$$
\left|\int_{R^{n}} f \cdot(g \cdot \nabla h) d x\right|=\left|\int_{R^{n}} \sum_{i=1}^{n} \sum_{j=1}^{n} g_{j} h_{i} \frac{\partial f_{i}}{\partial x_{j}} d x\right| \leq \int_{R^{n}}|g||h||\nabla f| d x .
$$


LEMMA 2.2. The following preliminary estimates are valid for problem (1-2):

$$
\begin{gathered}
|F[u \cdot \nabla u]| \leq|\xi|\|u(t)\|^{2}, \quad|\hat{p}| \leq\|u(t)\|^{2}, \\
\|p(t)\| \leq\|u(t)\|_{\infty}\|u(t)\|, \quad\|\Delta p(t)\| \leq C\|\Delta u(t)\|^{3 / 2}, \\
\left\|u_{t}(t)\right\| \leq\|u(t)\|_{\infty}\|\nabla u(t)\|+\|\Delta u(t)\|+\|\nabla p(t)\|, \\
\left\|p_{t}(t)\right\| \leq 2\|u(t)\|_{\infty}\left\|u_{t}(t)\right\| .
\end{gathered}
$$

Proof. Let $v_{0}=0$. Then $(v, q)=(0,0)$. By Lemma 2.1, we can get the estimates.

LEMmA 2.3. Let $u_{0}, v_{0} \in H^{2}, w_{0} \in L^{1}$. Let $(u, p),(v, q)$ be the solutions of problem (1-2) corresponding to $u_{0}, v_{0}$. Then

$$
\begin{aligned}
|\hat{w}| & \leq\left|\widehat{w_{0}}\right|+2|\xi| \int_{0}^{t}[\|u(s)\|+\|v(s)\|]\|w(s)\| d s \\
& \leq\left\|w_{0}\right\|_{L^{1}}+2|\xi| \int_{0}^{t}[\|u(s)\|+\|v(s)\|]\|w(s)\| d s .
\end{aligned}
$$

Proof. Applying the Fourier transform to the equations (3) yields

$$
\hat{w}_{t}+|\xi|^{2} \hat{w}+F[w \cdot \nabla u+v \cdot \nabla w+\nabla \pi]=0 .
$$

It follows easily that

$$
\left[\hat{w} e^{|\xi|^{2} t}\right]_{t}+F[w \cdot \nabla u+v \cdot \nabla w+\nabla \pi] e^{|\xi|^{2} t}=0 .
$$

Integrating in time gives

$$
\hat{w}=e^{-|\xi|^{2} t}\left[\widehat{w_{0}}-\int_{0}^{t} F[w \cdot \nabla u+v \cdot \nabla w+\nabla \pi](\xi, s) e^{|\xi|^{2} s} d s\right] .
$$

Therefore by using the first two estimates in Lemma 2.1, we obtain

$$
\begin{aligned}
|\hat{w}| & \leq\left|\widehat{w_{0}}\right|+\int_{0}^{t}|F[w \cdot \nabla u+v \cdot \nabla w+\nabla \pi]| d s \\
& \leq\left|\widehat{w_{0}}\right|+2|\xi| \int_{0}^{t}[\|u(s)\|+\|v(s)\|]\|w(s)\| d s \\
& \leq\left\|w_{0}\right\|_{L^{1}}+2|\xi| \int_{0}^{t}[\|u(s)\|+\|v(s)\|]\|w(s)\| d s .
\end{aligned}
$$

Lemma 2.4. Let $g=g(x, t), h=h(x, t) \in L^{\infty}\left(0, \infty ; H^{1}\left(R^{n}\right)\right)\left(x \in R^{n}, t \geq 0, n \geq 1\right)$ satisfy the energy inequality

$$
\begin{aligned}
& \frac{d}{d t}\left[(1+t)^{l} \int_{R^{n}}|\hat{g}|^{2} d \xi\right]+(1+t)^{l} \int_{R^{n}}|\xi|^{2}|\hat{g}|^{2} \xi \\
& \quad \leq C(1+t)^{l-1} \int_{R^{n}}|\hat{g}|^{2} d \xi+C(1+t)^{k} \int_{R^{n}}|\hat{h}|^{2} d \xi
\end{aligned}
$$

where $k, l$ are integers. Let $B(t)=\left\{\left.\xi \in R^{n}|(1+t)| \xi\right|^{2} \leq C\right\}$. Then we have the estimate

$$
\frac{d}{d t}\left[(1+t)^{l} \int_{R^{n}}|\hat{g}|^{2} d \xi\right] \leq C(1+t)^{l-1} \int_{B(t)}|\hat{g}|^{2} d \xi+C(1+t)^{k} \int_{R^{n}}|\hat{h}|^{2} d \xi
$$


Proof. Since $R^{n}=B(t) \cup B(t)^{c}$, we get

$$
\begin{aligned}
& (1+t)^{l} \int_{R^{n}}|\xi|^{2}|\hat{g}|^{2} d \xi=(1+t)^{l} \int_{B(t)}|\xi|^{2}|\hat{g}|^{2} d \xi+(1+t)^{l} \int_{B(t)^{c}}|\xi|^{2}|\hat{g}|^{2} d \xi \\
& \geq C(1+t)^{l-1} \int_{B(t)^{c}}|\hat{g}|^{2} d \xi=C(1+t)^{l-1} \int_{R^{n}}|\hat{g}|^{2} d \xi-C(1+t)^{l-1} \int_{B(t)}|\hat{g}|^{2} d \xi
\end{aligned}
$$

Substituting this inequality into the original one, the lemma is proved.

LEMmA 2.5 (Gagliardo-Nirenberg's inequality). For all $1 \leq p, q, r \leq \infty$ and for all integers $n \geq 1, m>k \geq 0$, there exist two constants $k / m \leq \alpha \leq 1, C>0$ such that for all $u \in C_{0}^{\infty}\left(R^{n}\right)$

$$
\begin{gathered}
\left\|D^{k} u\right\|_{L^{p}} \leq C\left\|D^{m} u\right\|_{L^{r}}^{\alpha}\|u\|_{L^{q}}^{1-\alpha}, \\
n / p-k=\alpha(n / r-m)+(1-\alpha) n / q, \\
\left\|D_{u}^{k}\right\|_{L^{p}}^{p}=\sum_{\beta_{1}+\cdots+\beta_{n}=k}\left\|\frac{\partial^{\beta_{1}+\cdots+\beta_{n}} u}{\partial x_{1}^{\beta_{1}} \cdots \partial x_{n}^{\beta_{n}}}\right\|_{L^{p}}^{p} .
\end{gathered}
$$

The only exception is that $\alpha \neq 1$ if $m-n / r=k, 1<r<\infty$.

Lemma 2.6 (Generalized Gronwall's inequality). Let $f(t), g(t) \geq 0, h(t) \geq 0$ satisfy the inequality

$$
g(t) \leq f(t)+\int_{0}^{t} g(s) h(s) d s, \quad \text { for any } 0 \leq t<\infty,
$$

where $h(t)$ satisfies $\int_{0}^{\infty} h(t) d t<\infty$. Then we have the estimate

$$
g(t) \leq f(t) \exp \left[\int_{0}^{\infty} h(t) d t\right], \quad \text { for any } 0 \leq t<\infty .
$$

3. Decay of solutions of problem (1-2), the case $\beta=0$. Before we begin our main work on problem (3-4), we need to establish some decay estimates on problem (1-2) with $u_{0} \in H^{2}$. These results are new.

The proof of Theorem 2 , the case $\beta=0$ will be given by the following three lemmas.

Lemma 3.1. Let $u_{0} \in H^{2}$. Then we have the following estimates:

$$
\sup _{0 \leq t<\infty}\|u(t)\|^{2} \leq\left\|u_{0}\right\|^{2}, \quad 2 \int_{0}^{\infty}\|\nabla u(t)\|^{2} d t \leq\left\|u_{0}\right\|^{2}, \quad \lim _{t \rightarrow \infty}\left[t\|\nabla u(t)\|^{2}\right]=0 .
$$

Proof. Forming the scalar product of (1) and the vector $2 u$ and integrating in the space $R^{n}$, we get

$$
\frac{d}{d t}\|u(t)\|^{2}+2\|\nabla u(t)\|^{2}=0
$$

It follows from this identity that (15) is correct. 
Discussions given below are restricted to the 2-dimensional case. Let $A=u_{1 x_{2}}-u_{2 x_{1}}$. Since $\nabla \cdot u=0$, we get

$$
A_{t}-\Delta A+\sum_{i=1}^{2} u_{i} A_{x_{i}}=0, \quad A(x, 0)=u_{01 x_{2}}-u_{02 x_{1}} .
$$

Making the scalar product of this equation and $2 A$, integrating in the space $R^{2}$, we get

$$
\frac{d}{d t}\|A(t)\|^{2}+2\|\nabla A(t)\|^{2}=0
$$

It is obvious that $\|\nabla u(t)\|^{2}=\|A(t)\|^{2},\|\Delta u(t)\|^{2}=\|\nabla A(t)\|^{2}$. Thus we have

$$
\frac{d}{d t}\|\nabla u(t)\|^{2}+2\|\Delta u(t)\|^{2}=0 \text {. }
$$

Therefore we obtain

$$
\sup _{0 \leq t<\infty}\|\nabla u(t)\|^{2} \leq\left\|\nabla u_{0}\right\|^{2}, \quad 2 \int_{0}^{\infty}\|\Delta u(t)\|^{2} d t \leq\left\|\nabla u_{0}\right\|^{2} .
$$

Moreover we observe that

$$
\frac{d}{d t}\|\nabla u(t)\|^{2}=-2\|\Delta u(t)\|^{2} \in L^{1}[0, \infty)
$$

Thus $\lim _{t \rightarrow \infty}\|\nabla u(t)\|^{2}$ exists.

On the other hand, $\|\nabla u(t)\|^{2} \in L^{1}[0, \infty)$. Thus $\lim _{t \rightarrow \infty}\|\nabla u(t)\|^{2}=0$. Because $\|\nabla u(t)\|^{2}$ is monotone decreasing, if $0<s<t<\infty$, we have

$$
\int_{s}^{\infty}\|\nabla u(\tau)\|^{2} d \tau \geq \int_{s}^{t}\|\nabla u(\tau)\|^{2} d \tau \geq(t-s)\|\nabla u(t)\|^{2} .
$$

Thus we obtain

$$
\int_{s}^{\infty}\|\nabla u(\tau)\|^{2} d \tau \geq \limsup _{t \rightarrow \infty}\left[(t-s)\|\nabla u(t)\|^{2}\right]=\limsup _{t \rightarrow \infty}\left[t\|\nabla u(t)\|^{2}\right]
$$

If we choose $s$ large enough, the quantity in the left-hand side can be made as small as desired. Therefore

$$
\lim _{t \rightarrow \infty}\left[t\|\nabla u(t)\|^{2}\right]=0 .
$$

LEMma 3.2. Let $u$ be the solution of problem (1-2) corresponding to the initial data $u_{0} \in H^{2}$. Then

$$
\|\Delta u(t)\| \leq C(1+t)^{-1} .
$$

Proof. If we form the scalar product of Eqs. (1) and $2 \Delta^{2} u$ and integrate in the space $R^{2}$, we get

$$
\frac{d}{d t}\|\Delta u(t)\|^{2}+2\|\nabla \Delta u(t)\|^{2}=2 \int_{R^{2}} \nabla \Delta u \cdot \nabla(u \cdot \nabla u) d x
$$

where

$$
\int_{R^{2}} \Delta^{2} u \cdot \nabla p d x=0
$$


We have the following simplifications:

$$
\begin{aligned}
2 \int_{R^{2}} \nabla \Delta u \cdot \nabla(u \cdot \nabla u) d x & \leq \frac{1}{2}\|\nabla \Delta u(t)\|^{2}+2 \int_{R^{2}}|\nabla(u \cdot \nabla u)|^{2} d x \\
2 \int_{R^{2}}|\nabla(u \cdot \nabla u)|^{2} d x & \leq C \int_{R^{2}}|\nabla u|^{4} d x+C\|u(t)\|_{\infty}^{2}\|\Delta u(t)\|^{2} \\
& \leq C\|\nabla u(t)\|_{\infty}^{2}\|\nabla u(t)\|^{2}+C\|u(t)\|_{\infty}^{2}\|\Delta u(t)\|^{2} \\
& \leq C\|u(t)\|\|\Delta u(t)\|\|\nabla u(t)\|\|\nabla \Delta u(t)\| \\
& \leq \frac{1}{2}\|\nabla \Delta u(t)\|^{2}+C\|u(t)\|^{2}\|\nabla u(t)\|^{2}\|\Delta u(t)\|^{2} .
\end{aligned}
$$

Therefore we get

$$
\frac{d}{d t}\|\Delta u(t)\|^{2}+\|\nabla \Delta u(t)\|^{2} \leq C\|u(t)\|^{2}\|\nabla u(t)\|^{2}\|\Delta u(t)\|^{2} .
$$

Applying Parseval's identity to the last energy inequality yields

$$
\frac{d}{d t} \int_{R^{2}}|\widehat{\Delta u}|^{2} d \xi+\int_{R^{2}}|\xi|^{2}|\widehat{\Delta u}|^{2} d \xi \leq C\|u(t)\|^{2}\|\nabla u(t)\|^{2} \int_{R^{2}}|\widehat{\Delta u}|^{2} d \xi,
$$

or we have the inequality

$$
\begin{aligned}
& \frac{d}{d t}\left[(1+t)^{3} \int_{R^{2}}|\widehat{\Delta u}|^{2} d \xi\right]+(1+t)^{3} \int_{R^{2}}|\xi|^{2}|\widehat{\Delta u}|^{2} d \xi \\
& \quad \leq 3(1+t)^{2} \int_{R^{2}}|\widehat{\Delta u}|^{2} d \xi+C(1+t)^{3}\|u(t)\|^{2}\|\nabla u(t)\|^{2} \int_{R^{2}}|\widehat{\Delta u}|^{2} d \xi \\
& \quad \leq C(1+t)^{2} \int_{R^{2}}|\widehat{\Delta u}|^{2} d \xi
\end{aligned}
$$

where we have used the fact that

$$
\lim _{t \rightarrow \infty}\left[(1+t)\|\nabla u(t)\|^{2}\right]=0 .
$$

More clearly, we have

$$
\frac{d}{d t}\left[(1+t)^{3} \int_{R^{2}}|\widehat{\Delta u}|^{2} d \xi\right]+(1+t)^{3} \int_{R^{2}}|\xi|^{2}|\widehat{\Delta u}|^{2} d \xi \leq C(1+t)^{2} \int_{R^{2}}|\widehat{\Delta u}|^{2} d \xi .
$$

By means of Lemma 2.4, we now obtain

$$
\begin{aligned}
& \frac{d}{d t}\left[(1+t)^{3} \int_{R^{2}}|\widehat{\Delta u}|^{2} d \xi\right] \leq C(1+t)^{2} \int_{B(t)}|\widehat{\Delta u}|^{2} d \xi \\
& \quad \leq C(1+t)^{2} \int_{B(t)}|\xi|^{4}|\hat{u}|^{2} d \xi \leq C \int_{B(t)}|\hat{u}|^{2} d \xi \leq C \int_{R^{2}}|\hat{u}|^{2} d \xi \leq C .
\end{aligned}
$$

Integrating in time $t$ yields

$$
(1+t)^{3} \int_{R^{2}}|\widehat{\Delta u}|^{2} d \xi \leq \int_{R^{2}}\left|\widehat{\Delta u_{0}}\right|^{2} d \xi+C t
$$

or we get

$$
\int_{R^{2}}|\widehat{\Delta u}|^{2} d \xi \leq C(1+t)^{-2}
$$


LEMmA 3.3. Let $(u, p)$ be the solutions of problem (1-2) corresponding to the initial velocity $u_{0} \in H^{2}$. Then

$$
\lim _{t \rightarrow \infty}\left[(1+t)\|p(t)\|^{2}+(1+t)^{2}\|\nabla p(t)\|^{2}\right]=0 .
$$

Proof. It can be proved by using the estimates in Lemma 2.2 that

$$
\begin{gathered}
\|p(t)\| \leq\|u(t)\|_{\infty}\|u(t)\|, \quad\|\Delta p(t)\| \leq C\|\Delta u(t)\|^{3 / 2}, \\
\|\nabla p(t)\|^{2} \leq\|p(t)\|\|\Delta p(t)\| .
\end{gathered}
$$

LEMmA 3.4. Let $(u, p)$ be the solution of problem (1-2) corresponding to the initial velocity $u_{0} \in H^{2}$. Then $\beta=0$ in Theorem 3 .

Proof. By using Lemma 2.2, it is very easy to prove.

If $u_{0}$ has more regularity, then we can demonstrate the following.

LEMMA 3.5. Let $u$ be the solution of problem (1-2) corresponding to the initial data $u_{0} \in H^{3}$. Then $\beta=0$ in Theorem 3 .

Proof. If we form the scalar product of Eqs. (1) and $2 \Delta^{3} u$ and integrate in the space $R^{2}$, we get

$$
\frac{d}{d t}\|\nabla \Delta u(t)\|^{2}+2\left\|\Delta^{2} u(t)\right\|^{2}=2 \int_{R^{2}} \Delta^{2} u \cdot \Delta(u \cdot \nabla u) d x .
$$

We have the following simplifications:

$$
\begin{aligned}
2 \int_{R^{2}} \Delta^{2} u \cdot \Delta(u \cdot \nabla u) d x & \leq\left\|\Delta^{2} u(t)\right\|^{2}+\int_{R^{2}}|\Delta(u \cdot \nabla u)|^{2} d x, \\
\int_{R^{2}}|\Delta(u \cdot \nabla u)|^{2} d x & \leq C \int_{R^{2}}|\Delta u|^{2}|\nabla u|^{2} d x+C \int_{R^{2}}|u|^{2}|\nabla \Delta u|^{2} d x \\
& \leq C\|\nabla u(t)\|_{\infty}^{2}\|\Delta u(t)\|^{2}+C\|u(t)\|_{\infty}^{2}\|\nabla \Delta u(t)\|^{2} \\
& \leq C\|\nabla u(t)\|\|\nabla \Delta u(t)\|\|\Delta u(t)\|^{2}+C\|u(t)\|\|\Delta u(t)\|\|\nabla \Delta u(t)\|^{2} \\
& \leq C\|\nabla u(t)\|^{2}\|\nabla \Delta u(t)\|^{2}+C\|u(t)\|\|\Delta u(t)\|\|\nabla \Delta u(t)\|^{2} .
\end{aligned}
$$

The decay results of Lemmas 3.1 and 3.2 yield

$$
\int_{R^{2}}|\Delta(u \cdot \nabla u)|^{2} d x \leq C(1+t)^{-1}\|\nabla \Delta u(t)\|^{2} .
$$

Therefore we get

$$
\frac{d}{d t}\|\nabla \Delta u(t)\|^{2}+\left\|\Delta^{2} u(t)\right\|^{2} \leq C(1+t)^{-1}\|\nabla \Delta u(t)\|^{2} .
$$

Applying Parseval's identity to the last energy inequality yields

$$
\frac{d}{d t} \int_{R^{2}}|\widehat{\nabla \Delta u}|^{2} d \xi+\int_{R^{2}}|\xi|^{2}|\widehat{\nabla \Delta u}|^{2} d \xi \leq C(1+t)^{-1} \int_{R^{2}}|\widehat{\nabla \Delta u}|^{2} d \xi
$$


or we have the inequality

$$
\begin{aligned}
& \frac{d}{d t}\left[(1+t)^{4} \int_{R^{2}}|\widehat{\nabla \Delta u}|^{2} d \xi\right]+(1+t)^{4} \int_{R^{2}}|\xi|^{2}|\widehat{\nabla \Delta u}|^{2} d \xi \\
& \leq 4(1+t)^{3} \int_{R^{2}}|\widehat{\nabla \Delta u}|^{2} d \xi+C(1+t)^{3} \int_{R^{2}}|\widehat{\nabla \Delta u}|^{2} d \xi \\
& \quad \leq C(1+t)^{3} \int_{R^{2}}|\widehat{\nabla \Delta u}|^{2} d \xi
\end{aligned}
$$

By utilizing Lemma 2.4, we get

$$
\begin{aligned}
& \frac{d}{d t}\left[(1+t)^{4} \int_{R^{2}}|\widehat{\nabla \Delta u}|^{2} d \xi\right] \leq C(1+t)^{3} \int_{B(t)}|\widehat{\nabla \Delta u}|^{2} d \xi \\
& \quad \leq C(1+t)^{3} \int_{B(t)}|\xi|^{6}|\hat{u}|^{2} d \xi \leq C \int_{B(t)}|\hat{u}|^{2} d \xi \leq C \int_{R^{2}}|\hat{u}|^{2} d \xi \leq C .
\end{aligned}
$$

Integrating in time $t$ yields

$$
(1+t)^{4} \int_{R^{2}}|\widehat{\nabla \Delta u}|^{2} d \xi \leq \int_{R^{2}}\left|\widehat{\nabla \Delta u_{0}}\right|^{2} d \xi+C t
$$

or we get

$$
\int_{R^{2}}|\widehat{\nabla \Delta u}|^{2} d \xi \leq C(1+t)^{-3}
$$

By using Gagliardo-Nirenberg's interpolation inequalities, it is very easy to get the estimates

$$
\begin{gathered}
\|u(t)\|_{\infty}^{2} \leq\|u(t)\|\|\Delta u(t)\| \leq C(1+t)^{-1}, \\
\|\nabla u(t)\|_{\infty}^{2} \leq C\|\nabla u(t)\|\|\nabla \Delta u(t)\| \leq C(1+t)^{-2} .
\end{gathered}
$$

REMARK 1. The following decay estimates are correct if the initial data $u_{0} \in H^{\infty}=$ $\bigcap_{m=1}^{\infty} H^{m}$ :

$$
\left\|\Delta^{m} u(t)\right\|^{2} \leq C(1+t)^{-2 m}, \quad\left\|\nabla \Delta^{m} u(t)\right\|^{2} \leq C(1+t)^{-2 m-1},
$$

where $m \geq 0$ is an integer. In fact, we have the following more precise results:

$$
\lim _{t \rightarrow \infty}(1+t)^{2 m}\left\|\Delta^{m} u(t)\right\|^{2}=0, \quad \lim _{t \rightarrow \infty}(1+t)^{2 m+1}\left\|\nabla \Delta^{m} u(t)\right\|^{2}=0 .
$$

It is worth mentioning that neither of these results has been obtained before if $u_{0} \in H^{3}$.

4. Proofs of Theorems 1 and 3. With the aid of the elementary estimates, the decay estimates of the solutions of problem (1-2) with $u_{0} \in H^{2}$, we can now develop our proof for the main theorem step by step.

LEMmA 4.1. Let $(u, p),(v, q)$ be the solutions of problem (1-2) corresponding to $u_{0}, v_{0} \in$ $H^{2}$. Then we have the following estimates:

$$
\begin{aligned}
& \frac{d}{d t}\left[(1+t)^{3} \int_{R^{2}}|\hat{w}|^{2} d \xi\right] \leq C(1+t)^{2} \int_{B(t)}|\hat{w}|^{2} d \xi \\
& \frac{d}{d t}\left[(1+t)^{5} \int_{R^{2}}|\widehat{\Delta w}|^{2} d \xi\right] \leq C(1+t)^{2} \int_{R^{2}}|\hat{w}|^{2} d \xi
\end{aligned}
$$


where $B(t)=\left\{\left.\xi \in R^{2}|(1+t)| \xi\right|^{2} \leq C\right\}$.

Proof. If we form the scalar product of equations (3) and $2 w$ and integrate in the space $R^{2}$, we get

$$
\frac{d}{d t}\|w(t)\|^{2}+2\|\nabla w(t)\|^{2}=-2 \int_{R^{2}} w \cdot(w \cdot \nabla u) d x
$$

where

$$
\int_{R^{2}} w \cdot(v \cdot \nabla w) d x=0, \quad \int_{R^{2}} w \cdot \nabla \pi d x=0
$$

Taking the last estimate in Lemma 2.1 into account, we have the following simplifications:

$$
-2 \int_{R^{2}} w \cdot(w \cdot \nabla u) d x \leq 2\|u(t)\|_{\infty}\|w(t)\|\|\nabla w(t)\| \leq\|u(t)\|_{\infty}^{2}\|w(t)\|^{2}+\|\nabla w(t)\|^{2} .
$$

Therefore we get

$$
\frac{d}{d t}\|w(t)\|^{2}+2\|\nabla w(t)\|^{2} \leq\|u(t)\|_{\infty}^{2}\|w(t)\|^{2}+\|\nabla w(t)\|^{2},
$$

or we have the simpler inequality

$$
\frac{d}{d t}\|w(t)\|^{2}+\|\nabla w(t)\|^{2} \leq\|u(t)\|_{\infty}^{2}\|w(t)\|^{2} .
$$

The right-hand side of the above estimate does not consist of $v$ or $v_{0}$; hence the constants appearing in any further arguments relating to this inequality have nothing to do with $v_{0}$.

By means of Lemmas 3.1 and 3.2, we get

$$
\|u(t)\|_{\infty}^{2} \leq\|u(t)\|\|\Delta u(t)\| \leq C(1+t)^{-1} .
$$

Therefore we obtain the estimate

$$
\frac{d}{d t}\|w(t)\|^{2}+\|\nabla w(t)\|^{2} \leq C(1+t)^{-1}\|w(t)\|^{2} .
$$

Moreover, applying Parseval's identity to the last energy inequality yields

$$
\frac{d}{d t} \int_{R^{2}}|\hat{w}|^{2} d \xi+\int_{R^{2}}|\xi|^{2}|\hat{w}|^{2} d \xi \leq C(1+t)^{-1} \int_{R^{2}}|\hat{w}|^{2} d \xi
$$

Obviously the following relation is valid:

$$
\begin{aligned}
& \frac{d}{d t}\left[(1+t)^{3} \int_{R^{2}}|\hat{w}|^{2} d \xi\right]+(1+t)^{3} \int_{R^{2}}|\xi|^{3}|\hat{w}|^{2} d \xi \\
& \quad \leq 3(1+t)^{2} \int_{R^{2}}|\hat{w}|^{2} d \xi+C(1+t)^{2} \int_{R^{2}}|\hat{w}|^{2} d \xi \leq C(1+t)^{2} \int_{R^{2}}|\hat{w}|^{2} d \xi
\end{aligned}
$$

By virtue of Lemma 2.4, we get

$$
\frac{d}{d t}\left[(1+t)^{3} \int_{R^{2}}|\hat{w}|^{2} d \xi\right] \leq C(1+t)^{2} \int_{B(t)}|\hat{w}|^{2} d \xi .
$$


If we form the scalar product of Eqs. (3) and $2 \Delta^{2} w$ and integrate in the space $R^{2}$, we get

$$
\begin{gathered}
\frac{d}{d t}\|\Delta w(t)\|^{2}+2\|\nabla \Delta w(t)\|^{2}=2 \int_{R^{2}} \nabla \Delta w \cdot \nabla(w \cdot \nabla u+v \cdot \nabla w) d x \\
\leq\|\nabla \Delta w(t)\|^{2}+\|\nabla(w \cdot \nabla u+v \cdot \nabla w)(t)\|^{2}
\end{gathered}
$$

thus we have

$$
\frac{d}{d t}\|\Delta w(t)\|^{2}+\|\nabla \Delta w(t)\|^{2} \leq\|\nabla(w \cdot \nabla u+v \cdot \nabla w)(t)\|^{2} .
$$

We have the following simplifications:

$$
\begin{aligned}
&\|\nabla(w \cdot \nabla u+v \cdot \nabla w)(t)\|^{2} \leq C \int_{R^{2}}|\nabla u|^{2}|\nabla w|^{2} d x+C \int_{R^{2}}|\nabla v|^{2}|\nabla w|^{2} d x \\
&+C\|w(t)\|_{\infty}^{2}\|\Delta u(t)\|^{2}+C\|v(t)\|_{\infty}^{2}\|\Delta w(t)\|^{2} \\
& C \int_{R^{2}}|\nabla u|^{2}|\nabla w|^{2} d x \leq C\|\nabla u(t)\|_{L^{4}}^{2}\|\nabla w(t)\|_{L^{4}}^{2} \\
& \leq C\|u(t)\|^{1 / 2}\|\Delta u(t)\|^{3 / 2}\|w(t)\|^{1 / 2}\|\Delta w(t)\|^{3 / 2} \\
& \leq C\|\Delta u(t)\|\|\Delta w(t)\|^{2}+C\|u(t)\|^{2}\|\Delta u(t)\|^{3}\|w(t)\|^{2} .
\end{aligned}
$$

Similarly, we have

$$
C \int_{R^{2}}|\nabla v|^{2}|\nabla w|^{2} d x \leq C\|\Delta v(t)\|\|\Delta w(t)\|^{2}+C\|v(t)\|^{2}\|\Delta v(t)\|^{3}\|w(t)\|^{2} .
$$

We also have the estimates

$$
\begin{aligned}
C\|w(t)\|_{\infty}^{2}\|\Delta u(t)\|^{2} & \leq C\|w(t)\|\|\Delta w(t)\|\|\Delta u(t)\|^{2} \\
& \leq C\|\Delta u(t)\|\|\Delta w(t)\|^{2}+C\|\Delta u(t)\|^{3}\|w(t)\|^{2}, \\
C\|v(t)\|_{\infty}^{2}\|\Delta w(t)\|^{2} & \leq C\|v(t)\|\|\Delta v(t)\|\|\Delta w(t)\|^{2} .
\end{aligned}
$$

We now have the estimate

$$
\begin{aligned}
\frac{d}{d t} \| \Delta & w(t)\left\|^{2}+\right\| \nabla \Delta w(t) \|^{2} \\
\leq & C[\|\Delta u(t)\|+\|\Delta v(t)\|+\|v(t)\|\|\Delta v(t)\|]\|\Delta w(t)\|^{2} \\
& +C\left[\|u(t)\|^{2}\|\Delta u(t)\|^{3}+\|v(t)\|^{2}\|\Delta v(t)\|^{3}+\|\Delta u(t)\|^{3}\right]\|w(t)\|^{2} .
\end{aligned}
$$

By using Lemmas 3.1 and 3.2, we obtain the inequality

$$
\begin{aligned}
& \frac{d}{d t}\|\Delta w(t)\|^{2}+\|\nabla \Delta w(t)\|^{2} \\
& \quad \leq C(1+t)^{-1}\|\Delta w(t)\|^{2}+C(1+t)^{-3}\|w(t)\|^{2} .
\end{aligned}
$$

Applying Parseval's identity to the last inequality yields

$$
\begin{aligned}
& \frac{d}{d t} \int_{R^{2}}|\widehat{\Delta w}|^{2} d \xi+\int_{R^{2}}|\xi|^{2}|\widehat{\Delta w}|^{2} d \xi \\
& \quad \leq C(1+t)^{-1} \int_{R^{2}}|\widehat{\Delta w}|^{2} d \xi+C(1+t)^{-3} \int_{R^{2}}|\hat{w}|^{2} d \xi
\end{aligned}
$$


Obviously the following relation is valid:

$$
\begin{aligned}
& \frac{d}{d t}\left[(1+t)^{5} \int_{R^{2}}|\widehat{\Delta w}|^{2} d \xi\right]+(1+t)^{5} \int_{R^{2}}|\xi|^{2}|\widehat{\Delta w}|^{2} d \xi \\
& \quad \leq 5(1+t)^{4} \int_{R^{2}}|\widehat{\Delta w}|^{2} d \xi+C(1+t)^{4} \int_{R^{2}}|\widehat{\Delta w}|^{2} d \xi+C(1+t)^{2} \int_{R^{2}}|\hat{w}|^{2} d \xi \\
& \quad \leq C(1+t)^{4} \int_{R^{2}}|\widehat{\Delta w}|^{2} d \xi+C(1+t)^{2} \int_{R^{2}}|\hat{w}|^{2} d \xi .
\end{aligned}
$$

By using Lemma 2.4, we now obtain

$$
\begin{aligned}
& \frac{d}{d t}\left[(1+t)^{5} \int_{R^{2}}|\widehat{\Delta w}|^{2} d \xi\right] \\
& \quad \leq C(1+t)^{4} \int_{B(t)}|\widehat{\Delta w}|^{2} d \xi+C(1+t)^{2} \int_{R^{2}}|\hat{w}|^{2} d \xi \\
& \quad=C(1+t)^{4} \int_{B(t)}|\xi|^{4}|\hat{w}|^{2} d \xi+C(1+t)^{2} \int_{R^{2}}|\hat{w}|^{2} d \xi \\
& \quad \leq C(1+t)^{2} \int_{B(t)}|\hat{w}|^{2} d \xi+C(1+t)^{2} \int_{R^{2}}|\hat{w}|^{2} d \xi \\
& \leq C(1+t)^{2} \int_{R^{2}}|\hat{w}|^{2} d \xi+C(1+t)^{2} \int_{R^{2}}|\hat{w}|^{2} d \xi
\end{aligned}
$$

Lemma 4.2. Proof of estimate (5) in Theorem 1.

Proof. By using the identity

$$
\frac{d}{d t}\|w(t)\|^{2}+2\|\nabla w(t)\|^{2}=-2 \int_{R^{2}} w \cdot(w \cdot \nabla u) d x
$$

we get

$$
\begin{aligned}
\frac{d}{d t}\|w(t)\|^{2}+2\|\nabla w(t)\|^{2} & \leq 2 \int_{R^{2}}|u||w||\nabla w| d x \leq 2\|u(t)\|_{L^{4}}\|w(t)\|_{L^{4}}\|\nabla w(t)\| \\
& \leq C\|u(t)\|^{1 / 2}\|\nabla u(t)\|^{1 / 2}\|w(t)\|^{1 / 2}\|\nabla w(t)\|^{3 / 2} \\
& \leq\|\nabla w(t)\|^{2}+C\|u(t)\|^{2}\|\nabla u(t)\|^{2}\|w(t)\|^{2}
\end{aligned}
$$

Integrating the above inequality in time $t$ gives

$$
\|w(t)\|^{2} \leq\left\|w_{0}\right\|^{2}+C \int_{0}^{t}\|u(s)\|^{2}\|\nabla u(s)\|^{2}\|w(s)\|^{2} d s .
$$

By using Gronwall's inequality and the estimate of Lemma 3.1, we obtain

$$
\|w(t)\|^{2} \leq\left\|w_{0}\right\|^{2} \exp \left[C \int_{0}^{\infty}\|u(t)\|^{2}\|\nabla u(t)\|^{2} d t\right] \leq\left\|w_{0}\right\|^{2} \exp \left[C\left\|u_{0}\right\|^{4}\right] .
$$

Thus

$$
\|w(t)\| \leq C\left\|w_{0}\right\|
$$

Up to now in this lemma, the constants are independent of $v_{0}$. This estimate also holds for periodic boundary value problems for (1). 
The estimate (20) in Lemma 4.1 yields

$$
\frac{d}{d t}\left[(1+t)^{5} \int_{R^{2}}|\widehat{\Delta w}|^{2} d \xi\right] \leq C(1+t)^{2} \int_{R^{2}}|\hat{w}|^{2} d \xi \leq C(1+t)^{2}\left\|w_{0}\right\|^{2} .
$$

Integrating in time $t$, we get

$$
(1+t)^{5} \int_{R^{2}}|\widehat{\Delta w}|^{2} d \xi \leq \int_{R^{2}}\left|\widehat{\Delta w_{0}}\right|^{2} d \xi+C(1+t)^{3}\left\|w_{0}\right\|^{2} .
$$

Thus one obtains

$$
\begin{gathered}
\int_{R^{2}}|\widehat{\Delta w}|^{2} d \xi \leq \int_{R^{2}}\left|\widehat{\Delta w_{0}}\right|^{2} d \xi(1+t)^{-5}+C\left\|w_{0}\right\|^{2}(1+t)^{-2} \\
\|\Delta w(t)\| \leq C(1+t)^{-1}\left\|w_{0}\right\|_{2}
\end{gathered}
$$

Further, by Gagliardo-Nirenberg's inequality (Lemma 2.5), we have

$$
\begin{aligned}
& \|w(t)\|_{\infty}^{2} \leq C\|w(t)\|\|\Delta w(t)\| \leq C(1+t)^{-1}\left\|w_{0}\right\|\left\|w_{0}\right\|_{2}, \\
& \|\nabla w(t)\|^{2} \leq C\|w(t)\|\|\Delta w(t)\| \leq C(1+t)^{-1}\left\|w_{0}\right\|\left\|w_{0}\right\|_{2} .
\end{aligned}
$$

Thus we get

$$
\|w(t)\|_{\infty} \leq C(1+t)^{-1 / 2}\left\|w_{0}\right\|_{2}, \quad\|\nabla w(t)\| \leq C(1+t)^{-1 / 2}\left\|w_{0}\right\|_{2} .
$$

Now we employ Lemma 2.1 to estimate $\left\|w_{t}(t)\right\|,\|\pi(t)\|,\|\nabla \pi(t)\|,\|\Delta \pi(t)\|,\|\pi(t)\|_{\infty}$, $\left\|\pi_{t}(t)\right\|$. It is very simple to obtain

$$
\begin{array}{cc}
\left\|w_{t}(t)\right\| \leq C(1+t)^{-1}\left\|w_{0}\right\|_{2}, & \left\|\pi_{t}(t)\right\| \leq C(1+t)^{-3 / 2}\left\|w_{0}\right\|_{2}, \\
\|\pi(t)\| \leq C(1+t)^{-1 / 2}\left\|w_{0}\right\|_{2}, & \|\nabla \pi(t)\| \leq C(1+t)^{-1}\left\|w_{0}\right\|_{2}, \\
\|\Delta \pi(t)\| \leq C(1+t)^{-3 / 2}\left\|w_{0}\right\|_{2}, & \|\pi(t)\|_{\infty} \leq C(1+t)^{-1}\left\|w_{0}\right\|_{2} .
\end{array}
$$

This lemma is very interesting. It illustrates that as long as the hypothesis $\left(u_{0}, A_{0}\right) \in$ $H^{2}$ is satisfied, which implies that $\left(u_{0}, A_{0}\right)$ decays very slowly as $|x| \rightarrow \infty$, and which guarantees that the global solutions of problem (1-2) exist and that $\|(u, A)(t)\|$, $\|(u, A)(t)\|_{\infty}$ decay to zero at a very slow rate, as $t \rightarrow \infty$, then the solutions of problem (1-2) are stable. We do not necessarily require that the initial velocity decay more rapidly, i.e., $\left(u_{0}, A_{0}\right) \in L^{r} \cap H^{2}, 1 \leq r<2$.

Corollary 1. Proof of the case $\beta=0$ in Theorem 3.

Proof. If $u_{0} \in H^{3}$, performing the operator $\nabla$ onto (1), we have

$$
\nabla u_{t}+\nabla(u \cdot \nabla u)-\nabla \Delta u+\nabla^{2} p=0
$$

and then

$$
\begin{aligned}
\left\|\nabla u_{t}(t)\right\| & \leq\|\nabla(u \cdot \nabla u)(t)\|+\|\nabla \Delta u(t)\|+\left\|\nabla^{2} p(t)\right\| \\
& \leq C\|\nabla u(t)\|_{\infty}\|\nabla u(t)\|+C\|u(t)\|_{\infty}\|\Delta u(t)\|+\|\nabla \Delta u(t)\|+C\|\Delta p(t)\| \\
& \leq C\|\nabla u(t)\|^{3 / 2}\|\nabla \Delta u(t)\|^{1 / 2}+C\|u(t)\|^{1 / 2}\|\Delta(t)\|^{3 / 2}+\|\nabla \Delta u(t)\|+C\|\Delta p(t)\| .
\end{aligned}
$$

By means of Lemmas 3.1-3.3 and 4.2, we get

$$
\left\|\nabla u_{t}(t)\right\| \leq C(1+t)^{-3 / 2} \text {. }
$$


Similar to what we did in Lemma 2.1, it is easy to get the estimate

$$
\left|\widehat{\nabla p_{t}}\right|^{2} \leq \sum_{i=1}^{2} \sum_{j=1}^{2}\left|F\left[\nabla\left(u_{i t} u_{j}+u_{i} u_{j t}\right)\right]\right|^{2} .
$$

Thus one gets

$$
\begin{aligned}
\left\|\nabla p_{t}(t)\right\|^{2}= & \left\|\widehat{\nabla p_{t}}(t)\right\|^{2} \leq \sum_{i=1}^{2} \sum_{j=1}^{2}\left\|F\left[\nabla\left(u_{i t} u_{j}+u_{i} u_{j t}\right)\right](t)\right\|^{2} \\
= & \sum_{i=1}^{2} \sum_{j=1}^{2}\left\|\nabla\left(u_{i t} u_{j}+u_{i} u_{j t}\right)(t)\right\|^{2} \\
\leq & C \sum_{i=1}^{2} \sum_{j=1}^{2}\left\{\left\|\nabla u_{i t}(t)\right\|^{2}\left\|u_{j}(t)\right\|_{\infty}^{2}+\left\|u_{i t}(t)\right\|^{2}\left\|\nabla u_{j}(t)\right\|_{\infty}^{2}\right. \\
& \left.\quad+\left\|\nabla u_{i}(t)\right\|_{\infty}^{2}\left\|u_{j t}(t)\right\|^{2}+\left\|u_{i}(t)\right\|_{\infty}^{2}\left\|\nabla u_{j t}(t)\right\|^{2}\right\} \\
\leq & C\left\|\nabla u_{t}(t)\right\|^{2}\|u(t)\|_{\infty}^{2}+C\left\|u_{t}(t)\right\|^{2}\|\nabla u(t)\|_{\infty}^{2} \\
\leq & C\|u(t)\|\|\Delta u(t)\|\left\|\nabla u_{t}(t)\right\|^{2} \\
& +C\|\nabla u(t)\|\|\nabla \Delta u(t)\|\left\|u_{t}(t)\right\|^{2} \leq C(1+t)^{-4}
\end{aligned}
$$

Lemma 4.3. Proof of estimate (6) in Theorem 1.

REMARK 2. If $u_{0} \in L^{r}$ or $v_{0} \in L^{r}, 1 \leq r<2$, then both $u_{0} \in L^{r}, v_{0} \in L^{r}$. This is because $w_{0} \in L^{1} \cap H^{2}$. Therefore we have

$$
\|u(t)\|+\|v(t)\| \leq C(1+t)^{1 / 2-1 / r} \leq C / \ln (e+t) .
$$

REMARK 3. If $u_{0}, v_{0} \in L^{1} \cap H^{2}$, then the assumptions are satisfied. In fact we have

$$
\|u(t)\|+\|v(t)\| \leq C(1+t)^{-1 / 2} .
$$

Proof. Using the estimate (19) of Lemma 4.1 and the estimate of Lemma 2.3, we have

$$
\begin{aligned}
& \frac{d}{d t}\left[(1+t)^{3} \int_{R^{2}}|\hat{w}|^{2} d \xi\right] \leq C(1+t)^{2} \int_{B(t)}|\hat{w}|^{2} d \xi \\
& \leq C(1+t)^{2} \int_{B(t)}\left\{\left\|w_{0}\right\|_{L^{1}}+2|\xi| \int_{0}^{t}\|w(s)\|[\|u(s)\|+\|v(s)\|] d s\right\}^{2} d \xi \\
& \leq C\left\|w_{0}\right\|_{L^{1}}^{2}(1+t)+C(1+t) \int_{0}^{t}\|w(t)\|^{2}\left[\|u(s)\|^{2}+\|v(s)\|^{2}\right] d s
\end{aligned}
$$

Integrating in time gives

$$
\begin{aligned}
(1+t)^{3} \int_{R^{2}}|\hat{w}|^{2} d \xi \leq & \int_{R^{2}}\left|\widehat{w_{0}}\right|^{2} d \xi \\
& +C\left\|w_{0}\right\|_{L^{1}}^{2}(1+t)^{2}+C(1+t)^{2} \int_{0}^{t}\|w(s)\|^{2}\left[\|u(s)\|^{2}+\|v(s)\|^{2}\right] d s
\end{aligned}
$$


or we get

$$
\begin{aligned}
(1+t) \int_{R^{2}}|\hat{w}|^{2} d \xi \leq & \int_{R^{2}}\left|\widehat{w_{0}}\right|^{2} d \xi+C\left\|w_{0}\right\|_{L^{1}}^{2} \\
& +C \int_{0}^{t}\|w(s)\|^{2}\left[\|u(s)\|^{2}+\|v(s)\|^{2}\right] d s \\
\leq & \int_{R^{2}}\left|\widehat{w}_{0}\right|^{2} d \xi+C\left\|w_{0}\right\|_{L^{1}}^{2}+C \int_{0}^{t}\|w(s)\|^{2}[\ln (e+s)]^{-2} d s
\end{aligned}
$$

Set

$$
g(t)=(1+t) \int_{R^{2}}|\hat{w}|^{2} d \xi, \quad h(t)=C(1+t)^{-1}[\ln (e+t)]^{-2} .
$$

By means of Gronwall's inequality, one obtains

$$
\begin{aligned}
&(1+t) \int_{R^{2}}|\hat{w}|^{2} d \xi \leq {\left[\int_{R^{2}}\left|\widehat{w_{0}}\right|^{2} d \xi+C\left\|w_{0}\right\|_{L^{1}}^{2}\right] } \\
& \times \exp \left[C \int_{0}^{\infty}(1+t)^{-1}[\ln (e+t)]^{-2} d t\right] \\
&\|w(t)\| \leq C(1+t)^{-1 / 2}\left\|w_{0}\right\|_{L} .
\end{aligned}
$$

In addition, using the estimate (20) of Lemma 4.1, we have the estimate

$$
\frac{d}{d t}\left[(1+t)^{5} \int_{R^{2}}|\widehat{\Delta w}|^{2} d \xi\right] \leq C(1+t)^{2} \int_{R^{2}}|\hat{w}|^{2} d \xi \leq C(1+t)\left\|w_{0}\right\|_{L}^{2}
$$

Integrating in time $t$, we obtain

$$
(1+t)^{5} \int_{R^{2}}|\widehat{\Delta w}|^{2} d \xi \leq \int_{R^{2}}\left|\widehat{\Delta w_{0}}\right|^{2} d \xi+C\left\|w_{0}\right\|_{L}^{2}(1+t)^{2},
$$

which is just the estimate

$$
\|\Delta w(t)\| \leq C\left\|w_{0}\right\|_{H}(1+t)^{-3 / 2} .
$$

As before, using Lemma 2.5, we get

$$
\begin{aligned}
\|w(t)\|_{\infty} & \leq C\|w(t)\|^{1 / 2}\|\Delta w(t)\|^{1 / 2} \leq C(1+t)^{-1}\left\|w_{0}\right\|_{H}, \\
\|\nabla w(t)\| & \leq C\|w(t)\|^{1 / 2}\|\Delta w(t)\|^{1 / 2} \leq C(1+t)^{-1}\left\|w_{0}\right\|_{H} .
\end{aligned}
$$

Further, by Lemma 2.1, we have

$$
\begin{array}{cc}
\|\pi(t)\| \leq C(1+t)^{-1}\left\|w_{0}\right\|_{H}, & \|\Delta \pi(t)\| \leq C(1+t)^{-2}\left\|w_{0}\right\|_{H}, \\
\|\pi(t)\|_{\infty} \leq C(1+t)^{-3 / 2}\left\|w_{0}\right\|_{H}, & \|\nabla \pi(t)\| \leq C(1+t)^{-3 / 2}\left\|w_{0}\right\|_{H}, \\
\left\|w_{t}(t)\right\| \leq C(1+t)^{-3 / 2}\left\|w_{0}\right\|_{H}, & \left\|\pi_{t}(t)\right\| \leq C(1+t)^{-2}\left\|w_{0}\right\|_{H} .
\end{array}
$$

Corollary 2. Let $u$ be the solution of problem (1-2) corresponding to the initial data $u_{0} \in H^{2}$. If $\|u(t)\| \leq C / \ln (e+t)$, then $\beta=1 / 2$ in Theorem 3 .

Lemma 4.4. Proof of estimate (8) in Theorem 1. 
Proof. There exists a constant $\delta>0$, such that

$$
\left|\widehat{w_{0}}\right| \leq C|\xi| \int_{R^{2}}|x|\left|w_{0}\right| d x \leq C|\xi|\left\|w_{0}\right\|_{W}, \quad \text { for }|\xi| \leq \delta .
$$

Using the estimates (21) and (23), we have

$$
\begin{aligned}
\frac{d}{d t}\left[(1+t)^{3} \int_{R^{2}}|\hat{w}|^{2} d \xi\right] & \leq C(1+t)^{2} \int_{B(t)}|\hat{w}|^{2} d \xi \\
& \leq C(1+t)^{2} \int_{B(t)}\left\{\left|\widehat{w_{0}}\right|+2|\xi| \int_{0}^{t}\|w(s)\|[\|u(s)\|+\|v(s)\|] d s\right\}^{2} d \xi \\
& \leq C\left\|w_{0}\right\|_{L^{1}}^{2}+C\left\{\int_{0}^{t}\|w(t)\|(\|u(s)\|+\|v(s)\|) d s\right\}^{2} \\
& \leq C\left\|w_{0}\right\|_{L^{1}}^{2}+C\left\|w_{0}\right\|^{2}[\ln (1+t)]^{2} .
\end{aligned}
$$

Integrating in time gives

$$
\begin{gathered}
(1+t)^{3} \int_{R^{2}}|\hat{w}|^{2} d \xi \leq \int_{R^{2}}\left|\widehat{w_{0}}\right|^{2} d \xi+C\left\|w_{0}\right\|_{L^{1}}^{2} t+C\left\|w_{0}\right\|_{L^{1}}^{2} t[\ln (1+t)]^{2} \\
\|w(t)\| \leq C(1+t)^{-1} \ln (1+t)\left\|w_{0}\right\|_{L}
\end{gathered}
$$

Iterating once more, i.e., using (21) and (23) again, we have

$$
\begin{aligned}
\frac{d}{d t}\left[(1+t)^{3} \int_{R^{2}}|\hat{w}|^{2} d \xi\right] & \leq C(1+t)^{2} \int_{B(t)}|\hat{w}|^{2} d \xi \\
& \leq C(1+t)^{2} \int_{B(t)}\left\{\left|\widehat{w_{0}}\right|+2|\xi| \int_{0}^{t}\|w(s)\|[\|u(s)\|+\|v(s)\|] d s\right\}^{2} d \xi \\
& \leq C\left\|w_{0}\right\|_{L^{1}}^{2}+C\left\{\int_{0}^{t}\|w(t)\|(\|u(s)\|+\|v(s)\|) d s\right\}^{2} \\
& \leq C\left\|w_{0}\right\|_{L^{1}}^{2}+C\left\|w_{0}\right\|^{2} .
\end{aligned}
$$

Integrating in time gives

$$
\begin{gathered}
(1+t)^{3} \int_{R^{2}}|\hat{w}|^{2} d \xi \leq \int_{R^{2}}\left|\widehat{w_{0}}\right|^{2} d \xi+C\left\|w_{0}\right\|_{L^{1}}^{2} t+C\left\|w_{0}\right\|_{L^{1}}^{2} t \\
\|w(t)\| \leq C(1+t)^{-1}\left\|w_{0}\right\|_{L} .
\end{gathered}
$$

Coupling the last estimate and estimate (22) yields

$$
\frac{d}{d t}\left[(1+t)^{5} \int_{R^{2}}|\widehat{\Delta w}|^{2} d \xi\right] \leq C(1+t)^{2} \int_{R^{2}}|\hat{w}|^{2} d \xi \leq C\left\|w_{0}\right\|^{2} .
$$

Integrating in time $t$ gives

$$
(1+t)^{5} \int_{R^{2}}|\widehat{\Delta w}|^{2} d \xi \leq \int_{R^{2}}\left|\widehat{\Delta w_{0}}\right|^{2} d \xi+C\left\|w_{0}\right\|^{2} t
$$

Therefore we have

$$
\|\Delta w(t)\| \leq C(1+t)^{-2} .
$$


Now it is very easy to get

$$
\begin{aligned}
& \|\nabla w(t)\| \leq C\left(\left\|w_{0}\right\|_{L^{1}}+\left\|w_{0}\right\|_{2}\right)(1+t)^{-1}, \\
& \|w(t)\|_{\infty} \leq C\left(\left\|w_{0}\right\|_{L^{1}}+\left\|w_{0}\right\|_{2}\right)(1+t)^{-1} .
\end{aligned}
$$

The other estimates follow lines that are by now familiar.

Corollary 3. Let the conditions of Lemma 4.4 be satisfied. Let $u$ be the solution of problem (1-2) corresponding to the initial data $u_{0} \in H^{2}$. Then $\beta=1$ in Theorem 3 .

Lemma 4.5. Let $u_{0}, v_{0} \in L^{1} \cap H^{2}, \int_{R^{2}} w_{0} d x \neq 0$. Then

$$
\begin{aligned}
& C_{5}\left|\int_{R^{2}} w_{0} d x\right|-\frac{C_{6} \ln (1+t)}{\sqrt{1+t}}\left\|w_{0}\right\|_{H} \leq(1+t)^{1 / 2}\|w(t)\| \leq C_{7}\left\|w_{0}\right\|_{H}, \\
& C_{8}\left|\int_{R^{2}} w_{0} d x\right|-\frac{C_{9} \ln (1+t)}{\sqrt{1+t}}\left\|w_{0}\right\|_{H} \leq(1+t)^{3 / 2}\|w(t)\| \leq C_{10}\left\|w_{0}\right\|_{H} .
\end{aligned}
$$

Proof. Let $y$ be the solution of the linear problem

$$
y_{t}-\Delta y=0, \quad y(x, 0)=w_{0}, \quad \nabla \cdot w_{0}=0 .
$$

Because $\nabla \cdot w_{0}=0, \hat{y}=\widehat{w_{0}} \exp \left[-|\xi|^{2} t\right]$, we get $\nabla \cdot y=0$.

Let $z=w-y$. Then $z$ satisfies the equations

$$
z_{t}+w \cdot \nabla u+v \cdot \nabla w-\Delta z+\nabla \pi=0, \quad \nabla \cdot z=0, \quad z(x, 0)=0 .
$$

If we form the scalar product of equations (3) and $2 z$ and integrate in the space $R^{2}$, we get

$$
\frac{d}{d t}\|z(t)\|^{2}+2\|\nabla z(t)\|^{2}=-2 \int_{R^{2}} z(w \cdot \nabla u+v \cdot \nabla w) d x
$$

where

$$
\int_{R^{2}} z \cdot \nabla \pi d x=0
$$

We have the following simplifications

$$
\begin{aligned}
-2 \int_{R^{2}} z \cdot(w \cdot \nabla u) d x & \leq 2\|u(t)\|_{\infty}\|w(t)\|\|\nabla z(t)\| \\
& \leq 2\|u(t)\|_{\infty}^{2}\|w(t)\|^{2}+\frac{1}{2}\|\nabla z(t)\|^{2}, \\
-2 \int_{R^{2}} z \cdot(v \cdot \nabla w) d x & \leq 2\|v(t)\|_{\infty}\|w(t)\|\|\nabla z(t)\| \\
& \leq 2\|v(t)\|_{\infty}^{2}\|w(t)\|^{2}+\frac{1}{2}\|\nabla z(t)\|^{2} .
\end{aligned}
$$

Therefore we get

$$
\frac{d}{d t}\|z(t)\|^{2}+\|\nabla z(t)\|^{2} \leq 2\left[\|u(t)\|_{\infty}^{2}+\|v(t)\|_{\infty}^{2}\right]\|w(t)\|^{2} .
$$

Applying Parseval's identity to the last energy inequality yields

$$
\frac{d}{d t} \int_{R^{2}}|\hat{z}|^{2} d \xi+\int_{R^{2}}|\xi|^{2}|\hat{z}|^{2} d \xi \leq 2\left[\|u(t)\|_{\infty}^{2}+\|v(t)\|_{\infty}^{2}\right] \int_{R^{2}}|\hat{w}|^{2} d \xi
$$


Obviously the following relation is valid:

$$
\begin{aligned}
& \frac{d}{d t}\left[(1+t)^{3} \int_{R^{2}}|\hat{z}|^{2} d \xi\right]+(1+t)^{3} \int_{R^{2}}|\xi|^{2}|\hat{z}|^{2} d \xi \\
& \quad \leq 3(1+t)^{2} \int_{R^{2}}|\hat{z}|^{2} d \xi+2(1+t)^{3}\left[\|u(t)\|_{\infty}^{2}+\|v(t)\|_{\infty}^{2}\right] \int_{R^{2}}|\hat{w}|^{2} d \xi \\
& \quad \leq 3(1+t)^{2} \int_{R^{2}}|\hat{z}|^{2} d \xi+C(1+t)^{2} \int_{R^{2}}|\hat{w}|^{2} d \xi \leq C(1+t)^{2} \int_{R^{2}}|\hat{w}|^{2} d \xi
\end{aligned}
$$

where we have used the fact $\|u(t)\|_{\infty}^{2}+\|v(t)\|_{\infty}^{2} \leq C(1+t)^{-1}$.

By using Lemma 2.4, we now obtain

$$
\begin{aligned}
\frac{d}{d t}\left[(1+t)^{3} \int_{R^{2}}|\hat{z}|^{2} d \xi\right] & \leq C(1+t)^{2} \int_{B(t)}|\hat{z}|^{2} d \xi \\
& \leq C(1+t)^{2} \int_{B(t)}\left\{2|\xi| \int_{0}^{t}\|w(s)\|[\|u(s)\|+\|v(s)\|] d s\right\}^{2} d \xi \\
& \leq C\left(\left\|w_{0}\right\|_{L^{1}}+\left\|w_{0}\right\|\right)^{2}[\ln (1+t)]^{2}
\end{aligned}
$$

where we have used the following estimates:

$$
\begin{gathered}
\hat{z}=-\int_{0}^{t} F[w \cdot \nabla u+v \cdot \nabla w+\nabla \pi] e^{-|\xi|^{2}(t-s)} d s \\
|\hat{z}| \leq 2|\xi| \int_{0}^{t}[\|u(s)\|+\|v(s)\|]\|w(s)\| d s .
\end{gathered}
$$

Integrating in time gives

$$
\begin{gathered}
(1+t)^{3} \int_{R^{2}}|\hat{z}|^{2} d \xi \leq C\left\|w_{0}\right\|_{L}^{2} t[\ln (1+t)]^{2} \\
\|z(t)\| \leq C\left\|w_{0}\right\|_{L}^{2}(1+t)^{-1} \ln (1+t) .
\end{gathered}
$$

Let $2 A=\left|\int_{R^{2}} w_{0} d x\right|$. Then $A>0$, and there exists a constant $\delta>0$, such that for any $\xi \in R^{2}$, if $|\xi| \leq \delta$, then $\left|\widehat{w_{0}}\right| \geq A$.

Now we have the estimate for the solution of the linear problem (26):

$$
\begin{aligned}
\int_{R^{2}}|y|^{2} d x & =\int_{R^{2}}|\hat{y}|^{2} d \xi=\int_{R^{2}}\left|\widehat{w_{0}}\right|^{2} e^{-2|\xi|^{2} t} d \xi \\
& \geq \int_{R^{2}}\left|\widehat{w_{0}}\right|^{2} e^{-2|\xi|^{2}(1+t)} d \xi \geq \int_{|\xi| \leq \delta}\left|\widehat{w_{0}}\right|^{2} e^{-2|\xi|^{2}(1+t)} d \xi \\
& \geq A^{2} \int_{|\xi| \leq \delta} e^{-2|\xi|^{2}(1+t)} d \xi \geq A^{2} \int_{0}^{2 \pi} \int_{0}^{\delta} r e^{-2 r^{2}(1+t)} d r d \theta \geq C A^{2}(1+t)^{-1} .
\end{aligned}
$$

Finally we have

$$
\begin{aligned}
\|w(t)\| \geq & \|y(t)\|-\|w(t)-y(t)\| \geq C A(1+t)^{-1 / 2} \\
& -C\left\|w_{0}\right\|_{L}(1+t)^{-1} \ln (1+t) \geq C(1+t)^{-1 / 2}
\end{aligned}
$$

for sufficiently large $t>0$. 
Using the same techniques as we displayed in the proof of the first estimate can complete the proof of the second one. The proof is omitted.

REMARK 4. If there exists $0<C_{0} \leq 1$ such that

$$
\begin{gathered}
\left|\int_{R^{2}} w_{0} d x\right| \geq C_{0} \int_{R^{2}}\left|w_{0}\right| d x>0 \\
\frac{C}{(1+t)^{1 / 2}} \int_{R^{2}}\left|w_{0}\right| d x-\frac{C \ln (1+t)}{1+t}\left(\left\|w_{0}\right\|_{L^{1}}+\left\|w_{0}\right\|\right) \geq C(1+t)^{-1 / 2},
\end{gathered}
$$

for all $t>0$, then we get

$$
C\left\|w_{0}\right\|_{L}(1+t)^{-1 / 2} \leq\|w(t)\| \leq C\left\|w_{0}\right\|_{L}(1+t)^{-1 / 2} .
$$

Combining the Lemmas 4.1-4.5, we have proved Theorem 1. Proposition 1 and Corollaries $1-3$ prove Theorem 2 .

5. $n(\geq 3)$-dimensional problems. We are concerned with $L^{2}$ and $L^{p}(p>n \geq 3)$ uniform stability for solutions to the Cauchy problem for $n$-dimensional incompressible Navier-Stokes equations (1-2). Let us state some relevant known results.

LEMma 5.1. Let $n \geq 3, u_{0} \in L^{2}$. Then there is a weak solution $u \in L^{\infty}\left(0, \infty ; L^{2}\right) \cap$ $L^{2}\left(0, T ; H^{1}\right)$, where $T>0$ is any finite constant. If $u_{0} \in L^{1} \cap L^{2}$, then the problem (1-2) admits a weak solution $u \in L^{\infty}\left(0, \infty ; L^{2}\right) \cap L^{2}\left(0, \infty ; H^{1}\right)$, and

$$
\|u(t)\| \leq C(1+t)^{-n / 4} \text {. }
$$

Furthermore, if $\int_{R^{n}} u_{0}(x) d x=0, \int_{R^{n}}|x|\left|u_{0}(x)\right| d x<\infty$, then

$$
\|u(t)\| \leq C(1+t)^{-1 / 2-n / 4}
$$

If $u_{0} \in L^{2} \cap L^{p}, p>n$, then there exists a constant $C>0$, such that if $\left\|u_{0}\right\|^{2(p-n) /(p(n-2))}\left\|u_{0}\right\|_{L^{p}} \leq C$, problem (1-2) has a unique global solution $u \in$ $L^{2}\left(0, \infty ; H^{1}\right) \cap C\left(0, \infty ; L^{2} \cap L^{p}\right)$. See $[H, K, K M, S 2, S e 1, S e 2, T, W, W 1, V]$.

LEMmA 5.2. If $u_{0} \in L^{2} \cap L^{p}, u \in L^{q}\left(0, \infty ; L^{p}\right)$, for some $p>n \geq 3, n / p+2 / q=1$, then $u \in L^{\infty}\left(0, \infty ; L^{p}\right),\|u(t)\|_{L^{p}}^{p} \leq C(1+t)^{-(p-2) n / 4}$. See [W2, V].

Lemma 5.3. If $u_{0}, v_{0} \in L^{2} \cap L^{p}, u \in L^{q}\left(0, \infty ; L^{p}\right)$, for some $p>n \geq 3, n / p+2 / q=1$, then there exists a constant $\delta=\delta(n, p)>0$, such that if $\left\|u_{0}-v_{0}\right\|_{L^{p}}<\delta$, a strong solution $v$ exists with $v \in L^{r}\left(0, \infty ; L^{p}\right), 4 p /(n(p-2))<r \leq \infty$. See [W2].

Lemma 5.4. If $u_{0} \in L^{1} \cap L^{p}, p>n,(1+|x|) u_{0}(x) \in L^{1}, \int_{R^{n}} u_{0}(x) d x=0$, then $\|u(t)\|_{\infty}=O\left(t^{-(n+1) / 2}\right)$. See [W3].

LEMMA 5.5. Let $(u, p)$ and $(v, q)$ be the weak solutions of the $n(\geq 3)$-dimensional problem (1-2) corresponding to the initial velocities $u_{0}$ and $v_{0}$. Let $(w, \pi)=(u-v, p-q)$. If $u_{0} \in L^{2} \cap L^{p}, u \in L^{q}\left(0, \infty ; L^{p}\right)$, for some $p>n \geq 3, n / p+2 / q=1$, then $u \in L^{\infty}\left(0, \infty ; L^{p}\right)$. If $v_{0} \in L^{2}$, we have the uniform stability estimate

$$
\|w(t)\| \leq C\left\|w_{0}\right\|, \quad \int_{0}^{\infty}\|\nabla w(t)\|^{2} d t \leq C\left\|w_{0}\right\|^{2}, \quad\|\hat{\pi}(t)\|_{\infty} \leq C\left\|w_{0}\right\|,
$$

where the constant $C$ depends only on the $L^{q}\left(0, \infty ; L^{p}\right)$ norm of $u$. 
Proof. The assertion $u \in L^{\infty}\left(0, \infty ; L^{p}\right)$ was proved by Verga [V]. By using the identity

$$
\frac{d}{d t}\|w(t)\|^{2}+2\|\nabla w(t)\|^{2}=-2 \int_{R^{n}} w \cdot(w \cdot \nabla u) d x
$$

we get

$$
\begin{aligned}
\frac{d}{d t}\|w(t)\|^{2}+2\|\nabla w(t)\|^{2} & \leq 2 \int_{R^{n}}|u||w||\nabla w| d x \leq 2\|u(t)\|_{L^{p}}\|w(t)\|_{L^{l}}\|\nabla w(t)\| \\
& \leq C\|u(t)\|_{L^{p}}\|w(t)\|^{1-\alpha}\|\nabla w(t)\|^{1+\alpha} \\
& \leq\|\nabla w(t)\|^{2}+C\|u(t)\|_{L^{p}}^{2 /(1-\alpha)}\|w(t)\|^{2}
\end{aligned}
$$

where $p>n \geq 3, l>2,1 / p+1 / l=1 / 2,0<\alpha=n / 2-n / l=n / p<1$. Since $n / p+2 / q=1$, so $2 / q=1-n / p=1-\alpha$, so $q=2 /(1-\alpha)$. Therefore

$$
\|u(t)\|_{L^{p}}^{2 /(1-\alpha)}=\|u(t)\|_{L^{p}}^{q} \in L^{1}[0, \infty) .
$$

Integrating the above inequality in time $t$ gives

$$
\|w(t)\|^{2}+\int_{0}^{t}\|\nabla w(s)\|^{2} d s \leq\left\|w_{0}\right\|^{2}+C \int_{0}^{t}\|u(s)\|_{L^{p}}^{q}\|w(s)\|^{2} d s .
$$

By using Gronwall's inequality and the estimate of Lemma 3.1, we obtain

$$
\|w(t)\|^{2}+\int_{0}^{t}\|\nabla w(s)\|^{2} d s \leq\left\|w_{0}\right\|^{2} \exp \left[C \int_{0}^{\infty}\|u(t)\|_{L^{p}}^{q} d t\right] .
$$

Thus

$$
\|w(t)\| \leq C\left\|w_{0}\right\|, \quad \int_{0}^{\infty}\|\nabla w(t)\|^{2} d t \leq C\left\|w_{0}\right\|^{2} .
$$

The last estimate follows immediately from the inequality from Lemma 2.1

$$
|\hat{\pi}| \leq[\|u(t)\|+\|v(t)\|]\|w(t)\| .
$$

The last estimate in Theorem 2 follows immediately from Wiegner's results [W3], Lemma 5.5 and the inequality from Lemma 2.1

$$
\|\pi(t)\| \leq\left[\|u(t)\|_{\infty}+\|v(t)\|_{\infty}\right]\|w(t)\| .
$$

LemMA 5.6. If $u_{0}, v_{0} \in L^{2} \cap L^{p}, u \in L^{q}\left(0, \infty ; L^{p}\right)$, for some $p>n \geq 3, n / p+2 / q=1$, then there exists a constant $\delta>0$, such that if $\left\|u_{0}-v_{0}\right\|_{L^{p}}<\delta$, a strong solution $v$ exists with $v \in L^{r}\left(0, \infty ; L^{p}\right), 4 p /(n(p-2))<r \leq \infty$, and

$$
\begin{gathered}
\sup _{\left\|u_{0}-v_{0}\right\|_{L^{p}}<\delta} \int_{0}^{\infty}\|v(t)\|_{L^{p}}^{q} d t<\infty, \\
\|u(t)-v(t)\|_{L^{p}} \leq C\left\|u_{0}-v_{0}\right\|_{L^{p}}, \\
\frac{p}{2} \int_{0}^{\infty} \int_{R^{n}}|u-v|^{p-2}|\nabla(u-v)|^{2} d x d t \\
+\frac{2(p-2)}{p} \int_{0}^{\infty} \int_{R^{n}}\left|\nabla\left(|u-v|^{p / 2}\right)\right|^{2} d x d t \leq C\left\|u_{0}-v_{0}\right\|_{L^{p}}^{p},
\end{gathered}
$$

where $C$ depends on the norm $\int_{0}^{\infty}\|u(t)\|_{L^{p}}^{q} d t$. 
Proof. The first assertion was proved by Wiegner [W2]. For the estimates, let $\| u_{0}-$ $v_{0} \|_{L^{p}}<\delta$. Because $4 p /(n(p-2))<2 p /(p-n)=q$,

$$
\|v(t)\|_{L^{p}}^{q} \leq C(1+t)^{-(p-2) n /(2(p-n))},
$$

and $(p-2) n /(2(p-n))>1$; so

$$
\int_{0}^{\infty}\|v(t)\|_{L^{p}}^{q} d t \leq C\left(n, p,\left\|v_{0}\right\|,\left\|v_{0}\right\|_{L^{p}}\right) \leq C\left(n, p,\left\|u_{0}\right\|,\left\|u_{0}\right\|_{L^{p}}\right) .
$$

Hence

$$
\sup _{\left\|u_{0}-v_{0}\right\|_{L^{p}}<\delta} \int_{0}^{\infty}\|v(t)\|_{L^{p}}^{q} d t \leq C\left(n, p,\left\|u_{0}\right\|,\left\|u_{0}\right\|_{L^{p}}\right)<\infty .
$$

If we form the scalar product of equations (3) and $p|w|^{p-1} w$ and integrate in the space $R^{n}$, we get

$$
\begin{aligned}
& \frac{d}{d t} \int_{R^{n}}|w|^{p} d x+p \int_{R^{n}}|w|^{p-2}|\nabla w|^{2} d x+\frac{4(p-2)}{p} \int_{R^{n}}\left|\nabla\left(|w|^{p / 2}\right)\right|^{2} d x \\
& \quad=-p \int_{R^{n}}\left(w \cdot \nabla u,|w|^{p-2} w\right) d x-p \int_{R^{n}}\left(v \cdot \nabla w,|w|^{p-2} w\right) d x-p \int_{R^{n}}\left(\nabla \pi,|w|^{p-2} w\right) d x
\end{aligned}
$$

where

$$
\begin{aligned}
\int_{R^{n}}\left(v \cdot \nabla w,|w|^{p-2} w\right) d x=0 \\
-\int_{R^{n}}\left(w \cdot \nabla u,|w|^{p-2} w\right) d x \\
=-\sum_{i=1}^{n} \sum_{j=1}^{n} \int_{R^{n}}|w|^{p-2} w_{i} \frac{\partial}{\partial x_{j}}\left(u_{i} w_{j}\right) d x \\
=\sum_{i=1}^{n} \sum_{j=1}^{n} \int_{R^{n}} u_{i} w_{j} \frac{\partial}{\partial x_{j}}\left(|w|^{p-2} w_{i}\right) d x \\
=\sum_{i=1}^{n} \sum_{j=1}^{n} \int_{R^{n}}|w|^{p-2} u_{i} w_{j} \frac{\partial w_{i}}{\partial x_{j}} d x+(p-2) \sum_{i=1}^{n} \sum_{j=1}^{n} \int_{R^{n}}|w|^{p-4} u_{i} w_{i} w_{j}\left(w, \frac{\partial w}{\partial x_{j}}\right) d x \\
\leq \int_{R^{n}}|w|^{p-2}\left(\sum_{i=1}^{n} \sum_{j=1}^{n}\left|u_{i} w_{j}\right|^{2}\right)^{1 / 2}\left(\sum_{i=1}^{n} \sum_{j=1}^{n}\left|\frac{\partial w_{i}}{\partial x_{j}}\right|^{2}\right)^{1 / 2} d x \\
\quad+(p-2) \int_{R^{n}}|w|^{p-3}\left(\sum_{i=1}^{n}\left|u_{i} w_{i}\right|\right)\left(\sum_{j=1}^{n}\left|w_{j}\right|\left|\frac{\partial w}{\partial x_{j}}\right|\right) d x \\
\leq(p-1) \int_{R^{n}}|u||w|^{p-1}|\nabla w| d x \leq \frac{1}{4} \int_{R^{n}}|w|^{p-2}|\nabla w|^{2} d x+(p-1)^{2} \int_{R^{n}}|u|^{2}|w|^{p} d x,
\end{aligned}
$$




$$
\begin{aligned}
p(p-1)^{2} \int_{R^{n}}|u|^{2}|w|^{p} d x \leq p & (p-1)^{2}\|u(t)\|_{L^{p}}^{2}\|w(t)\|_{L^{p^{2} /(p-2)}}^{p} \\
& =p(p-1)^{2}\|u(t)\|_{L^{p}}^{2}\|\phi(t)\|_{L^{2 p /(p-2)}}^{2} \quad\left(\text { where } \phi=|w|^{p / 2}\right) \\
& \leq C\|u(t)\|_{L^{p}}^{2}\|\phi(t)\|^{2(1-n / p)}\|\nabla \phi(t)\|^{2 n / p} \\
& \leq C\|u(t)\|_{L^{p}}^{2 p /(p-n)}\|\phi(t)\|^{2}+\frac{p-2}{p}\|\nabla \phi(t)\|^{2} \\
& =C\|u(t)\|_{L^{p}}^{q}\|\phi(t)\|^{2}+\frac{p-2}{p}\|\nabla \phi(t)\|^{2} \\
& =C\|u(t)\|_{L^{p}}^{q}\|w(t)\|_{L^{p}}^{p}+\frac{p-2}{p} \int_{R^{n}}\left|\nabla\left(|w|^{p / 2}\right)\right|^{2} d x
\end{aligned}
$$

$-\int_{R^{n}}\left(\nabla \pi,|w|^{p-2} w\right) d x=\sum_{i=1}^{n} \int_{R^{n}} \pi \frac{\partial}{\partial x_{i}}\left(|w|^{p-2} w_{i}\right) d x$

$$
\begin{aligned}
& =(p-2) \sum_{i=1}^{n} \int_{R^{n}} \pi w_{i}|w|^{p-4}\left(w, \frac{\partial w}{\partial x_{i}}\right) d x \\
& \leq(p-2) \int_{R^{n}}|\pi||w|^{p-2}|\nabla w| d x \\
& \leq \frac{1}{4} \int_{R^{n}}|w|^{p-2}|\nabla w|^{2} d x+(p-2)^{2} \int_{R^{n}}|\pi|^{2}|w|^{p-2} d x .
\end{aligned}
$$

Since

$$
\Delta \pi=-\nabla(w \cdot \nabla u+v \cdot \nabla w)=-\sum_{i=1}^{n} \sum_{j=1}^{n} \frac{\partial^{2}}{\partial x_{i} \partial x_{j}}\left(u_{i} w_{j}+v_{i} w_{j}\right)
$$

by Calderon-Zygmund's inequality [St], one has

$$
\|\pi(t)\|_{L^{r}}^{2} \leq C(n, r) \sum_{i=1}^{n} \sum_{j=1}^{n}\left\|\left(u_{i} w_{j}+v_{i} w_{j}\right)(t)\right\|_{L^{r}}^{2}, \quad 1<r<\infty
$$

thus

$$
\begin{aligned}
p(p- & 2)^{2} \int_{R^{n}}|\pi|^{2}|w|^{p-2} d x \\
& \leq p(p-2)^{2}\|\pi(t)\|_{L^{p^{2} /(2(p-1))}}^{2}\|w(t)\|_{L^{p^{2} /(p-2)}}^{p-2} \\
& \leq C\left[\|u(t)\|_{L^{p}}^{2}+\|v(t)\|_{L^{p}}^{2}\right]\|w(t)\|_{L^{p^{2} /(p-2)}}^{2}\|w(t)\|_{L^{p^{2} /(p-2)}}^{p-2} \\
& =C\left[\|u(t)\|_{L^{p}}^{2}+\|v(t)\|_{L^{p}}^{2}\right]\|\phi(t)\|_{L^{2 p /(p-2)}}^{2} \quad\left(\text { where } \phi=|w|^{p / 2}\right) \\
& \leq C\left[\|u(t)\|_{L^{p}}^{2}+\|v(t)\|_{L^{p}}^{2}\right]\|\phi(t)\|^{2(1-n / p)}\|\nabla \phi(t)\|^{2 n / p} \\
& \leq C\left[\|u(t)\|_{L^{p}}^{2 p /(p-n)}+\|v(t)\|_{L^{p}}^{2 p /(p-n)}\right]\|\phi(t)\|^{2}+\frac{p-2}{p}\|\nabla \phi(t)\|^{2} \\
& =C\left[\|u(t)\|_{L^{p}}^{q}+\|v(t)\|_{L^{p}}^{q}\right]\|\phi(t)\|^{2}+\frac{p-2}{p}\|\nabla \phi(t)\|^{2} \\
& =C\left[\|u(t)\|_{L^{p}}^{q}+\|v(t)\|_{L^{p}}^{q}\right]\|w(t)\|_{L^{p}}^{p}+\frac{p-2}{p} \int_{R^{n}}\left|\nabla\left(|w|^{p / 2}\right)\right|^{2} d x .
\end{aligned}
$$


Now Eq. (27) is simplified to the inequality

$$
\begin{aligned}
& \frac{d}{d t} \int_{R^{n}}|w|^{p} d x+\frac{p}{2} \int_{R^{n}}|w|^{p-2}|\nabla w|^{2} d x+\frac{2(p-2)}{p} \int_{R^{n}}\left|\nabla\left(|w|^{p / 2}\right)\right|^{2} d x \\
& \leq C\left[\|u(t)\|_{L^{p}}^{q}+\|v(t)\|_{L^{p}}^{q}\right]\|w(t)\|_{L^{p}}^{p} .
\end{aligned}
$$

According to Wiegner's result in [W3]

$$
\sup _{\left\|u_{0}-v_{0}\right\|_{L^{p}}<\delta} \int_{0}^{\infty}\|v(t)\|_{L^{p}}^{q} d t<\infty, \quad \int_{0}^{\infty}\left[\|u(t)\|_{L^{p}}^{q}+\|v(t)\|_{L^{p}}^{q}\right] d t \leq C<\infty .
$$

Gronwall's inequality yields the estimate

$$
\begin{gathered}
\int_{R^{n}}|w|^{p} d x+\frac{p}{2} \int_{0}^{t} \int_{R^{n}}|w|^{p-2}|\nabla w|^{2} d x d s+\frac{2(p-2)}{p} \int_{0}^{t} \int_{R^{n}}\left|\nabla\left(|w|^{p / 2}\right)\right|^{2} d x d s \\
\leq C \exp \left\{C \int_{0}^{\infty}\left[\|u(t)\|_{L^{p}}^{q}+\|v(t)\|_{L^{p}}^{q}\right] d t\right\} \int_{R^{n}}\left|w_{0}\right|^{p} d x,
\end{gathered}
$$

or

$$
\begin{gathered}
\|u(t)-v(t)\|_{L^{p}} \leq C\left\|u_{0}-v_{0}\right\|_{L^{p}} \\
\frac{p}{2} \int_{0}^{\infty} \int_{R^{n}}|w|^{p-2}|\nabla w|^{2} d x d t+\frac{2(p-2)}{p} \int_{0}^{\infty} \int_{R^{n}}\left|\nabla\left(|w|^{p / 2}\right)\right|^{2} d x d t \leq C\left\|u_{0}-v_{0}\right\|_{L^{p}}^{p}
\end{gathered}
$$

Estimate (11) in Theorem 2 can be proved by combining estimate (10) and Theorem 4 , which is verified below.

If $v_{0}=0$, then $v(x, t)=0$. So we get

$$
\begin{gathered}
\|u(t)\|_{L^{p}} \leq C\left\|u_{0}\right\|_{L^{p}} \\
\left.\frac{p}{2} \int_{0}^{\infty} \int_{R^{n}}\left|u^{p-2}\right| \nabla u\right|^{2} d x d t+\frac{2(p-2)}{p} \int_{0}^{\infty} \int_{R^{n}}\left|\nabla\left(|u|^{p / 2}\right)\right|^{2} d x d t \leq C\left\|u_{0}\right\|_{L^{p}}^{p},
\end{gathered}
$$

where $C$ depends on the $L^{q}\left(0, \infty ; L^{p}\right)$ norm of $u$.

Let $u_{0} \in L^{2} \cap L^{p}, u \in L^{q}\left(0, \infty ; L^{p}\right)$, for some $p>n \geq 3, n / p+2 / q=1,1<m<$ $n /(n-2), q_{1}=2 m p /(m p-n), f=|u|^{p / 2}$. Then $n /(m p)+2 / q_{1}=1$, and

$$
\begin{gathered}
\|u(t)\|_{L^{m p}}=\|f(t)\|_{L^{2 m}}^{2 / p} \leq C\|f(t)\|^{(2 m-m n+n) /(m p)}\|\nabla f(t)\|^{(m n-n) /(m p)}, \\
\|u(t)\|_{L^{m p}}^{q_{1}} \leq C\|f(t)\|^{2(2 m-m n+n) /(m p-n)}\|\nabla f(t)\|^{2(m n-n) /(m p-n)} \\
\leq C\|f(t)\|^{2(2 m-m n+n) /(m(p-n))}+\|\nabla f(t)\|^{2} \\
=C\|u(t)\|_{L^{p}}^{p(2 m-m n+n) /(m(p-n))}+\int_{R^{n}}\left|\nabla\left(|u|^{p / 2}\right)\right|^{2} d x \\
\|u(t)\|_{L^{p}}^{p(2 m-m n+n) /(m(p-n))} \leq C(1+t)^{-(p-2)(2 m-m n+n) n /(4 m(p-n))}
\end{gathered}
$$

Here we can always choose $m$ so that $1<m<\frac{(p-2) n^{2}}{4(p-n)+(p-2)(n-2) n}<\frac{n}{n-2}$, which is equivalent to $(p-2)(2 m-m n+n) n /(4 m(p-n))>1$. For example $m=n^{2} /\left(n^{2}-2 n+4\right)>$ 
1 , which is independent of $p$. So

$$
\begin{gathered}
\|u(t)\|_{L^{p}}^{p(2 m-m n+n) /(m(p-n))} \in L^{1}[0, \infty), \\
\int_{R^{n}}\left|\nabla\left(|u|^{p / 2}\right)\right|^{2} d x \in L^{1}[0, \infty), \\
\|u(t)\|_{L^{m p}}^{q_{1}} \in L^{1}[0, \infty), \\
u \in L^{q_{1}}\left[0, \infty ; L^{m p}\right), \quad u \in L^{\infty}\left[0, \infty ; L^{m p}\right),
\end{gathered}
$$

where $\frac{n}{m p}+\frac{2}{q_{1}}=1$. Iterating infinitely many times, noticing that $m>1, m^{k} \rightarrow \infty$, as $k \rightarrow \infty$, we have proved

Lemma 5.7. Let $u_{0} \in L^{2} \cap L^{p}, u \in L^{q}\left(0, \infty ; L^{p}\right)$, for some $p>n \geq 3, n / p+2 / q=1$. Then $u \in L^{q_{1}}\left(0, \infty ; L^{m p}\right) \cap L^{\infty}\left(0, \infty ; L^{m p}\right)$, where $m=n^{2} /\left(n^{2}-2 n+4\right)>1, m p>n \geq 3$, $n /(m p)+2 / q_{1}=1$. Therefore $u \in \bigcap_{k \geq 1}\left(L^{q_{k}}\left(0, \infty ; L^{m^{k} p}\right) \cap L^{\infty}\left(0, \infty ; L^{m^{k} p}\right)\right)$, where $n /\left(m^{k} p\right)+2 / q_{k}=1$.

Lemma 5.8. Let $u_{0} \in L^{2} \cap L^{p}, u \in L^{q}\left(0, \infty ; L^{p}\right)$, for some $p>n \geq 3, n / p+2 / q=1$. Then $u \in\left(\bigcap_{p \leq r<\infty} L^{\frac{2 r}{r-n}}\left(0, \infty ; L^{r}\right)\right) \cap\left(\bigcap_{2 \leq s<\infty} L^{\infty}\left(0, \infty ; L^{s}\right)\right)$.

Proof. From Lemmas 3.1 and 5.7, one knows that $u \in\left(L^{q_{k}}\left(0, \infty ; L^{m^{k}} p\right)\right) \cap\left(L^{\infty}(0, \infty\right.$; $\left.\left.L^{2} \cap L^{m^{k} p}\right)\right), q_{k}=\frac{2 m^{k} p}{m^{k} p-n}, k \geq 1$. Let $\alpha, \beta, \gamma$ be real numbers such that $p \leq \alpha \leq \beta \leq$ $\gamma<\infty, \alpha<\gamma$. Hölder's inequality yields the estimate

$$
\begin{gathered}
\|u(t)\|_{L^{\beta}} \leq\|u(t)\|_{L^{\alpha}}^{\alpha(\gamma-\beta) /(\beta(\gamma-\alpha))}\|u(t)\|_{L^{\gamma}}^{\gamma(\beta-\alpha) /(\beta(\gamma-\alpha))}, \\
\int_{0}^{\infty}\|u(t)\|_{L^{\beta}}^{2 \beta /(\beta-n)} d t \leq \int_{0}^{\infty}\|u(t)\|_{L^{\alpha}}^{2 \alpha(\gamma-\beta) /((\gamma-\alpha)(\beta-n))}\|u(t)\|_{L^{\gamma}}^{2 \gamma(\beta-\alpha) /((\gamma-\alpha)(\beta-n))} d t \\
\leq\left(\int_{0}^{\infty}\|u(t)\|_{L^{\alpha}}^{2 \alpha /(\alpha-n)} d t\right)^{(\gamma-\beta)(\alpha-n) /((\gamma-\alpha)(\beta-n))} \\
\times\left(\int_{0}^{\infty}\|u(t)\|_{L^{\gamma}}^{2 \gamma /(\gamma-n)} d t\right)^{(\beta-\alpha)(\gamma-n) /((\gamma-\alpha)(\beta-n))} .
\end{gathered}
$$

For any $r: m^{k} p \leq r \leq m^{k+1} p$, let $\alpha=m^{k} p, \beta=r, \gamma=m^{k+1} p$. Then

$$
\begin{aligned}
\|u(t)\|_{L^{r}} & =\|u(t)\|_{L^{\beta}} \leq\|u(t)\|_{L^{\alpha}}^{\alpha(\gamma-\beta) /(\beta(\gamma-\alpha))}\|u(t)\|_{L^{\gamma}}^{\gamma(\beta-\alpha) /(\beta(\gamma-\alpha))} \\
& =\|u(t)\|_{L^{m^{k} p}}^{m^{k} p\left(m^{k+1} p-r\right) /\left(r\left(m^{k+1} p-m^{k} p\right)\right)}\|u(t)\|_{L^{m^{k+1} p}}^{m^{k+1} p\left(r-m^{k} p\right) /\left(r\left(m^{k+1} p-m^{k} p\right)\right)}<\infty
\end{aligned}
$$




$$
\begin{aligned}
& \int_{0}^{\infty}\|u(t)\|_{L^{r}}^{2 r /(r-n)} d t=\int_{0}^{\infty}\|u(t)\|_{L^{\beta}}^{2 \beta /(\beta-n)} d t \\
& \leq\left(\int_{0}^{\infty}\|u(t)\|_{L^{\alpha}}^{2 \alpha /(\alpha-n)} d t\right)^{(\gamma-\beta)(\alpha-n) /((\gamma-\alpha)(\beta-n))} \\
& \quad \times\left(\int_{0}^{\infty}\|u(t)\|_{L^{\gamma}}^{2 \gamma /(\gamma-n)} d t\right)^{(\beta-\alpha)(\gamma-n) /((\gamma-\alpha)(\beta-n))} \\
& =\left(\int_{0}^{\infty}\|u(t)\|_{L^{m^{k}} p}^{2 m^{k} p /\left(m^{k} p-n\right)} d t\right)^{\left(m^{k+1} p-r\right)\left(m^{k} p-n\right) /\left(\left(m^{k+1} p-m^{k} p\right)(r-n)\right)} \\
& \quad \times\left(\int_{0}^{\infty}\|u(t)\|_{L^{m^{k+1}} p}^{2 m^{k+1} p /\left(m^{k+1} p-n\right)} d t\right)^{\left(r-m^{k} p\right)\left(m^{k+1} p-n\right) /\left(\left(m^{k+1} p-m^{k} p\right)(r-n)\right)}<\infty .
\end{aligned}
$$

For 2-dimensional Navier-Stokes equations, we studied the long time uniform stability and asymptotic behavior of the strong solutions. For the $n(\geq 3)$-dimensional problem, we investigated the regularity and long time uniform stability of the strong solutions. We have repeatedly assumed that $u_{0} \in L^{2} \cap L^{p}, u \in L^{q}\left(0, \infty ; L^{p}\right)$, for some $p>n \geq 3$, $n / p+2 / q=1$ to justify that $u$ has more regularity, namely

$$
u \in\left(\bigcap_{p \leq r<\infty} L^{\frac{2 r}{r-n}}\left(0, \infty ; L^{r}\right)\right) \cap\left(\bigcap_{2 \leq s<\infty} L^{\infty}\left(0, \infty ; L^{s}\right)\right) .
$$

A natural question is what space should $u_{0}$ belong to so that $u \in L^{q}\left(0, \infty ; L^{p}\right)$, for some $p>n \geq 3, n / p+2 / q=1$. Wiegner [W3] proved that if $u_{0} \in L^{1} \cap L^{p}, p>n$, $(1+|x|) u_{0}(x) \in L^{1}, \int_{R^{n}} u_{0}(x) d x=0$, then $\|u(t)\|_{\infty}=O\left(t^{-(n+1) / 2}\right)$. Also Lemma 5.1 shows that $\|u(t)\| \leq C(1+t)^{-1 / 2-n / 4}$. So

$$
\|u(t)\|_{L^{p}}^{q} \leq\left(\|u(t)\|_{\infty}^{p-2}\|u(t)\|^{2}\right)^{2 /(p-n)} \leq C(1+t)^{-(n+1)(p-1) /(p-n)} .
$$

It turns out that $u \in L^{q}\left(0, \infty ; L^{p}\right)$, for any $p>n \geq 3, n / p+2 / q=1$.

Another sufficient condition is that $u_{0}$ is small. This is well known.

\section{REFERENCES}

[CF] P. Constantin and C. Fefferman, Direction of vorticity and the problem of global regularity for the Navier-Stokes equations, Indiana University Mathematics Journal 42, 775-789 (1993)

[GZ] B. Guo and L. Zhang, Decay of solutions to magnetohydrodynamics equations in two space dimensions, Proceedings of the Royal Society of London Ser. A 449, 79-91 (1995)

[H] J. G. Heywood, The Navier-Stokes equations, on the existence, regularity and decay of solutions, Indiana University Mathematics Journal 29, 639-681 (1980)

[K] T. Kato, Strong $L^{p}$-solutions of the Navier-Stokes equations in $R^{m}$, with applications to weak solutions, Math. Z. 187, 471-480 (1984)

[KM] R. Kajikiya and T. Miyakawa, On $L^{2}$-decay of weak solutions of the Navier-Stokes equations in $R^{n}$, Math. Z. 192, 135-148 (1986)

[P] R. Pego, Stability in systems of conservation laws with dissipation, Lectures in Applied Math. 23, 345-357 (1986)

[PSW] R. Pego, P. Smereka, and M. I. Weinstein, Oscillatory instability of traveling waves for a KdVBurgers equation, Physica D 67, 45-65 (1993)

[W] F. Weissler, The Navier-Stokes initial value problems in $L^{p}$, Arch. Rational Mech. Analysis 74, 219-230 (1980)

[W1] M. Wiegner, Decay results for weak solutions of the Navier-Stokes equations on $R^{n}$, J. London Math. Soc. 35, 303-313 (1987) 
[W2] M. Wiegner, Decay and stability in $L^{p}$ for strong solutions of the Cauchy problem for the NavierStokes equations, in "The Navier-Stokes Equations, Theory and Numerical Methods", edited by J. G. Heywood et al, Springer-Verlag, New York, Lecture Notes in Math. 1431, 95-99 (1990)

[W3] M. Wiegner, Decay of the $L_{\infty}$-norm of solutions of the Navier-Stokes equations in unbounded domains, Acta Appl. Math. 37, 215-219 (1994)

[S] M. Schonbek, Decay of solutions of parabolic conservation laws, Comm. Partial Differential Equations 7, 449-473 (1980)

[S1] M. Schonbek, $L^{2}$ decay for weak solutions of the Navier-Stokes equations, Arch. Rational Mech. Analysis 88, 209-222 (1985)

[S2] M. Schonbek, Large time behavior of solutions to the Navier-Stokes equations, Comm. Partial Differential Equations 11, 733-763 (1986)

[Se] P. Secchi, $L^{2}$ stability for weak solutions of the Navier-Stokes equations in $R^{3}$, Indiana University Mathematics Journal 36, 685-691 (1987)

[Se1] J. Serrin, On the interior regularity of weak solutions of the Navier-Stokes equations, Arch. Rational Mech. Analysis 9, 187-195 (1962)

[Se2] J. Serrin, The initial value problems for the Navier-Stokes equations, in "Nonlinear Problems" (R. E. Langer, ed.), University of Wisconsin Press, Madison, 1963, pp. 69-98

[St] E. M. Stein, Singular integrals and differentiability properties of functions, Princeton University Press, 1970

[T] R. Temam, Navier-Stokes equations, theory and numerical analysis, North-Holland, Amsterdam and New York, 1979

[V] H. Beirao da Veiga, Existence and asymptotic behavior for strong solutions of the Navier-Stokes equations in the whole space, Indiana University Mathematics Journal 36, 149-166 (1987)

[VS] H. Beirao da Veiga and P. Secchi, $L^{p}$-stability for the strong solutions of the Navier-Stokes equations in the whole space, Arch. Rational Mech. Anal. 98, 65-69 (1987)

[Z1] L. Zhang, Initial value problem for a nonlinear parabolic equation with singular integraldifferential term, Acta Math. Appl. Sinica 8, 367-376 (1992)

[Z2] L. Zhang, Decay estimates for the solutions of some nonlinear evolution equations, J. Differential Equations 116, 31-58 (1995)

[Z3] L. Zhang, Sharp rate of decay of solutions to 2-dimensional Navier-Stokes equations, Comm. Partial Differential Equations 20, 119-127 (1995)

[Z4] L. Zhang, Decay estimates of solutions to the initial value problems for a generalized nonlinear Korteweg-de Vries equation, Chinese Ann. Math. 16A, 22-32 (1995) [in Chinese]

[ZG1] Y. Zhou and B. Guo, The periodic boundary value problem and the initial value problem for the generalized Korteweg-de Vries systems of higher order, Acta Math. Sinica 27, 154-176 (1984)

[ZG2] Y. Zhou and B. Guo, Initial value problems for a nonlinear singular integral-differential equation of deep water, in "Partial Differential Equations", edited by S. S. Chern, Springer-Verlag, New York, Lecture Notes in Math. 1306, 278-290 (1988) 